\title{
8. MIDDLE PLIOCENE PLANKTONIC AND BENTHIC FORAMINIFERS FROM THE SUBARCTIC NORTH PACIFIC: SITES 883 AND $887^{1}$
}

\author{
Harry J. Dowsett ${ }^{2}$ and Scott E. Ishman ${ }^{2}$
}

\begin{abstract}
Diverse planktonic and benthic foraminifer assemblages exhibiting fair to good preservation were recovered from the middle Pliocene interval of Sites 883 and 887 in the subarctic North Pacific Ocean. The middle Pliocene planktonic assemblage at Site 883 is similar to late Pleistocene and modern assemblages in the region and probably represents paleoceanographic conditions approximately the same as those of today. The benthic assemblage from Site 883 indicates a middle Pliocene deepening of the oxygen minima. At Site 887 the planktonic assemblage is indicative of surface conditions slightly warmer than those of today while the benthic assemblage indicates conditions approximately the same as those of today.
\end{abstract}

\section{INTRODUCTION}

For several years the U.S. Geological Survey has been documenting the magnitude and variability of middle Pliocene (3.15-2.85 Ma) climate as part of the PRISM (Pliocene Research, Interpretation, and Synoptic Mapping) Project (Cronin and Dowsett, 1993). Examination of middle Pliocene sedimentary sequences from the Northern and Southern hemispheres by PRISM and other researchers reveals signs of significant surface water warming, reduced sea-ice cover, reduced global ice volume, elevated sea levels, and significant changes in vegetation patterns relative to today (Dowsett and Cronin, 1990; Webb and Harwood, 1991; Barron, 1992; Dowsett et al., 1992; Cronin et al., 1993; Shackleton et al., in press; Dowsett et al., 1994; Willard, 1994).

Ocean Drilling Program (ODP) Leg 145 represents a unique opportunity to examine complete, high-resolution sequences containing open-ocean calcareous microfossils from the North Pacific and to better understand the nature of the subarctic during the middle Pliocene. In this paper, we document the nature and variability of the planktonic and benthic foraminifer faunas from Sites 883 and 887 in the northwest and northeast Pacific, respectively.

The PRISM time interval is 3.15 to $2.85 \mathrm{Ma}$ using the time scale of Berggren et al. (1985). Leg 145 workers have adopted the use of the Cande and Kent (1992) time scale, which places the PRISM time interval at 3.29-2.98 Ma. This interval is approximately coincident with the Kaena through Mammoth paleomagnetic subchrons.

\section{METHODS}

All samples were processed by first oven drying $\left(\leq 50^{\circ} \mathrm{C}\right)$ and then soaking in dilute calgon solution for several hours to disaggregate the sediment. Disaggregated sediment was washed through a $150 \mu \mathrm{m}$ mesh and oven dried at $\leq 50^{\circ} \mathrm{C}$. Several methods were developed to separate foraminifer tests from siliceous material that dominates all samples. Procedures using controlled air and water currents to separate foraminifers from diatoms did not result in significantly faster separation, so samples were picked for foraminifers manually. For planktonic foraminifers, samples were placed on large 60 -square micropaleontological picking trays, and foraminifers were removed from individual squares using a random number pattern until 300 individuals were obtained. In samples with less than 300 individuals, all planktonic foraminifers were obtained. For benthic foramin-

\footnotetext{
${ }^{1}$ Rea, D.K., Basov, I.A., Scholl, D.W., and Allan, J.F. (Eds.), 1995. Proc. ODP, Sci. Results, 145: College Station, TX (Ocean Drilling Program).

${ }^{2}$ College of Oceanic and Atmospheric Sciences, Oregon State University, Oceanography Admin. BIdg. 104, Corvallis, OR 97331-5503, U.S.A.
}

ifers, samples were scanned to determine which contained abundant benthic foraminifers and all benthic foraminifers were removed from those samples. The average number of benthic formaminifers obtained ranged from 150 to 250 .

Individuals were then fixed on a standard 60-square micropaleontological slide based upon their designation as species. For planktonic foraminifers, the taxonomies of Parker (1962, 1967), Blow (1969), and Kennett and Srinivasan (1983) were used whereas Loeblich and Tappan (1988) was used for the benthic foraminifers.

Scanning electron micrographs were obtained by coating specimens with $30 \AA$ of gold palladium and then viewing them on a JEOL JSM-35C. Specimens illustrated on Plates 1-3 are deposited at the U.S. National Museum in Washington D.C.

\section{SITE 883}

Site 883 is situated on the edge of the Detroit Seamount at $51^{\circ} 11.908^{\prime} \mathrm{N}$ and $167^{\circ} 46.128^{\prime} \mathrm{E}$ in $2384 \mathrm{~m}$ of water (Fig. 1). The core site is overlain by Pacific Deep Water and the surface Western Subarctic Gyre (Keigwin et al., 1992). Sea-surface temperatures average $1.5^{\circ} \mathrm{C}$ in February and reach $10.5^{\circ} \mathrm{C}$ in August (Schweitzer, 1993). Diatom and radiolarian biochronology and available magnetostratigraphy indicate that the PRISM time interval covers Cores $145-883 \mathrm{~B}$ $14 \mathrm{H}$ to $-16 \mathrm{H}$. A $170-\mathrm{cm}$ gap at the bottom of Core $145-883 \mathrm{~B}-15 \mathrm{H}$ can be correlated to the upper portion of Core $145-883 \mathrm{C}-16 \mathrm{H}$ using the Gamma-Ray Attenuation Porosity Evaluation (GRAPE) density records of the two holes. Thus, by combining Holes $883 \mathrm{~B}$ and $883 \mathrm{C}$, we have a fairly complete record covering the PRISM time slab (3.29$2.98 \mathrm{Ma})$ at a sample spacing of approximately $10 \mathrm{k} . \mathrm{y}$.

\section{Planktonic Foraminifers}

Planktonic foraminifers are common in most of the 49 samples examined from Site 883 (Tables 1 and 2). In all but 7 samples, more than 300 identifiable specimens were recovered. Preservation ranges from good to poor, with many samples showing signs of pervasive dissolution and recalcification of foraminifer tests.

The assemblage at Site 883 is dominated by Globigerina bulloides and cold-water Neogloboquadrina species, including $N$. pachyderma and $N$. atlantica. Other, less common, taxa include Globigerina umbilicata and Globigerinita glutinata. Rare occurrences of Orbulina universa, Globorotalia scitula, Globigerina incisa, and Turborotalita quinqueloba complete the assemblage. Neogloboquadrina pachyderma shows a preference for dextral coiling at Site 883 which along with other available age data suggests correlation to coiling interval CD15 of Lagoe and Thompson (1988). 
Table 1. Occurrences of planktonic foraminifers, Hole 883B.

\begin{tabular}{|c|c|c|c|c|c|c|c|c|c|c|c|c|c|c|c|}
\hline $\begin{array}{l}\text { Core, section, } \\
\text { interval }(\mathrm{cm})\end{array}$ & $\begin{array}{l}\text { Depth } \\
\text { (mbsf) }\end{array}$ & 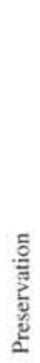 & 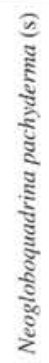 & 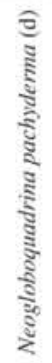 & 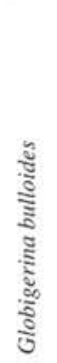 & 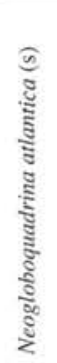 & 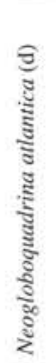 & 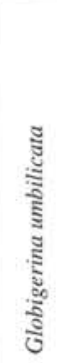 & 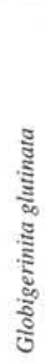 & 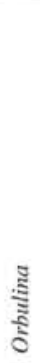 & 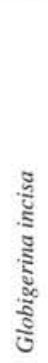 & 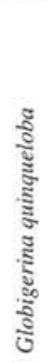 & 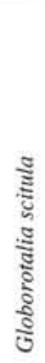 & 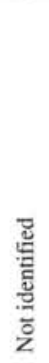 & ㄹّㅁ \\
\hline $14 \mathrm{H}-1,5-10$ & 121.95 & G & 10 & 6 & 86 & 20 & 28 & 135 & 18 & & & 20 & 1 & 4 & 328 \\
\hline $14 \mathrm{H}-1,45-50$ & 122.35 & $\mathrm{~F}$ & 1 & 42 & 45 & 84 & 70 & 10 & 7 & & 3 & & i & & 263 \\
\hline $14 \mathrm{H}-1,85-90$ & 122.75 & G & 1 & 83 & 24 & 57 & 96 & 8 & 5 & & & & 2 & 2 & 278 \\
\hline $14 \mathrm{H}-1,121-126$ & 123.11 & $\mathrm{~F}$ & 5 & 35 & 24 & 117 & 117 & 15 & 3 & & 5 & 3 & 2 & $i$ & 327 \\
\hline $14 \mathrm{H}-2,15-20$ & 123.55 & $\mathrm{~F}$ & 3 & 28 & 49 & 79 & 126 & 3 & 10 & & 2 & 1 & 1 & 1 & 303 \\
\hline $14 \mathrm{H}-2,55-60$ & 123.95 & $\mathrm{~F}$ & 4 & 3 & 20 & 44 & 44 & 12 & 9 & & 2 & 2 & & 4 & 144 \\
\hline $14 \mathrm{H}-2,90-95$ & 124.30 & $\mathrm{~F}$ & 1 & 20 & 46 & 100 & 122 & 18 & 18 & & 5 & & & 1 & 331 \\
\hline $14 \mathrm{H}-2,135-140$ & 124.75 & $\mathrm{~F}$ & 6 & 12 & 46 & 98 & 102 & 19 & 12 & & 3 & 6 & 1 & 3 & 308 \\
\hline $14 \mathrm{H}-3,25-30$ & 125.15 & $\mathrm{~F}$ & $\begin{array}{l}0 \\
3\end{array}$ & 14 & 98 & 75 & 59 & 29 & 15 & & 3 & 2 & 1 & 3 & 302 \\
\hline $14 \mathrm{H}-3,65-70$ & 125.55 & $\mathrm{~F}$ & 1 & 10 & 52 & 95 & 99 & 15 & 24 & & & 2 & 1 & 3 & 301 \\
\hline $14 \mathrm{H}-3,102-107$ & 125.92 & $\mathrm{~F}$ & & 23 & 171 & 16 & 41 & 12 & 22 & & & & 2 & & 287 \\
\hline $14 \mathrm{H}-4,35-40$ & 126.75 & P & 1 & 5 & 34 & 20 & 62 & 13 & 33 & & & & & 1 & 169 \\
\hline $14 \mathrm{H}-4,110-115$ & 127.50 & $\mathrm{~F}$ & 5 & & 63 & 34 & 30 & 22 & 7 & & & & 1 & & 162 \\
\hline $14 \mathrm{H}-5,45-50$ & 128.35 & $\mathrm{~F}$ & & 11 & 201 & 11 & 48 & 21 & 16 & 1 & & & i & & 310 \\
\hline $14 \mathrm{H}-5,125-130$ & 129.15 & $\mathrm{P}$ & 2 & 12 & 116 & 28 & 75 & 22 & 52 & & 1 & & & & 308 \\
\hline $14 \mathrm{H}-6,55-60$ & 129.95 & $\mathrm{P}$ & & 12 & 170 & 15 & 58 & 27 & 23 & & & & 5 & 1 & 311 \\
\hline $14 \mathrm{H}-6,136-141$ & 130.76 & $\mathrm{~F}$ & 1 & 17 & 98 & 36 & 104 & 42 & 24 & 2 & & & & 7 & 331 \\
\hline $14 \mathrm{H}-7,65-70$ & 131.55 & $\mathrm{~F}$ & & 9 & 185 & 13 & 44 & 78 & 16 & 2 & & & 2 & 1 & 350 \\
\hline $15 \mathrm{H}-1,45-50$ & 131.85 & $\mathrm{~F}$ & & 40 & 124 & 61 & 37 & 32 & 13 & & & & $i$ & & 308 \\
\hline $15 \mathrm{H}-2,15-20$ & 133.05 & G & 6 & 28 & 39 & 94 & 113 & 20 & 17 & & 6 & & i & 2 & 326 \\
\hline $15 \mathrm{H}-2,135-140$ & 134.25 & $\mathrm{~F}$ & 3 & 18 & 188 & 13 & 65 & 65 & 9 & & & I & i & & 363 \\
\hline $15 \mathrm{H}-3,25-30$ & 134.65 & $\mathrm{~F}$ & 2 & 19 & 71 & 96 & 85 & 16 & 15 & & 1 & & 1 & 1 & 307 \\
\hline $15 \mathrm{H}-3,102-107$ & 135.42 & $\mathrm{~F}$ & 4 & 9 & 78 & 112 & 84 & 20 & 2 & & 4 & & 7 & 6 & 326 \\
\hline $15 \mathrm{H}-4,35-40$ & 136.25 & $\mathrm{~F}$ & 3 & 5 & 105 & 64 & 81 & 28 & 17 & & 2 & 2 & 5 & 4 & 316 \\
\hline $15 \mathrm{H}-4,111-116$ & 137.01 & $\mathrm{~F}$ & i & 8 & 102 & 92 & 97 & 18 & 11 & & i & i & 3 & 5 & 339 \\
\hline $15 \mathrm{H}-5,46-5 \mathrm{I}$ & 137.86 & $\mathrm{~F}$ & $\mathrm{i}$ & 2 & 130 & 99 & 91 & 26 & i1 & & i & & $i$ & & 362 \\
\hline $15 \mathrm{H}-5,126-131$ & 138.66 & $\mathrm{~F}$ & i & i & 117 & 111 & 83 & 23 & 7 & 4 & & & 2 & 3 & 352 \\
\hline $15 \mathrm{H}-6,50-55$ & 139.40 & $\mathrm{~F}$ & i & 9 & 175 & 32 & 54 & 17 & 21 & & & & & 1 & 310 \\
\hline $15 \mathrm{H}-7,35-40$ & 140.25 & $\mathrm{~F}$ & $i$ & 6 & 87 & 67 & 99 & 17 & 28 & & & & 1 & i & 307 \\
\hline $16 \mathrm{H}-1,5-10$ & 140.95 & G & 1 & 7 & 203 & 11 & 42 & 42 & 3 & & 1 & & i & 1 & 312 \\
\hline $16 \mathrm{H}-1,85-90$ & 141.75 & $\mathrm{~F}$ & 1 & 10 & 193 & 35 & 13 & 32 & 8 & & 1 & & . & 3 & 295 \\
\hline $16 \mathrm{H}-2,15-20$ & 142.55 & $\mathrm{~F}$ & & & 77 & 127 & 84 & 16 & 9 & & 2 & & & 1 & 316 \\
\hline $16 \mathrm{H}-2,90-95$ & 143.30 & $\mathrm{~F}$ & & 1 & 242 & 17 & 15 & 52 & 4 & & & & & 1 & 332 \\
\hline $16 \mathrm{H}-3,25-30$ & 144.15 & $\mathrm{~F}$ & 2 & & 182 & 54 & 28 & 32 & 8 & & 2 & & & 5 & 313 \\
\hline $16 \mathrm{H}-3,101-106$ & 144.91 & $\mathrm{~F}$ & & 3 & 219 & 25 & 40 & 44 & 3 & & & & 1 & & 335 \\
\hline $16 \mathrm{H}-4,35-40$ & 145.75 & $\mathrm{~F}$ & 1 & 3 & 201 & 24 & 32 & 64 & 7 & & & & 2 & & 331 \\
\hline $16 \mathrm{H}-4,111-115$ & 146.51 & $\mathrm{~F}$ & 2 & 3 & 162 & 22 & 36 & 68 & 17 & 1 & 3 & & 2 & 1 & 317 \\
\hline $16 \mathrm{H}-5,45-50$ & 147.35 & $\mathrm{~F}$ & 4 & & 189 & 31 & 26 & 50 & 4 & & 3 & & $i$ & 3 & 311 \\
\hline $16 \mathrm{H}-5,120-125$ & 148.10 & $\mathrm{~F}$ & 3 & 3 & 163 & 27 & 33 & 72 & 7 & & & & 4 & 1 & 313 \\
\hline $16 \mathrm{H}-6,50-55$ & 148.90 & $\mathrm{~F}$ & 2 & 5 & 152 & 23 & 21 & 108 & 14 & & & & & & 325 \\
\hline $16 \mathrm{H}-6,135-140$ & 149.75 & $\mathrm{~F}$ & 1 & 1 & 145 & 27 & 19 & 111 & 4 & & & & & & 308 \\
\hline $16 \mathrm{H}-7,64-69$ & 150.54 & $\mathrm{~F}$ & & 1 & 143 & 34 & 22 & 55 & 43 & & & & 4 & 2 & 304 \\
\hline
\end{tabular}

Note: $\mathrm{G}=\operatorname{good}, \mathrm{F}=$ fair, and $\mathrm{P}=$ poor.

Table 2. Occurrences of planktonic foraminifers, Hole $883 \mathrm{C}$.

\begin{tabular}{|c|c|c|c|c|c|c|c|c|c|c|c|c|c|c|c|}
\hline $\begin{array}{l}\text { Core, section, } \\
\text { interval }(\mathrm{cm})\end{array}$ & $\begin{array}{l}\text { Depth } \\
\text { (mbsf) }\end{array}$ & 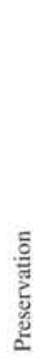 & 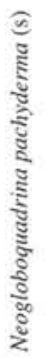 & 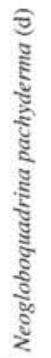 & 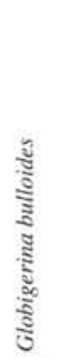 & 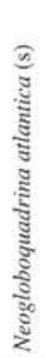 & 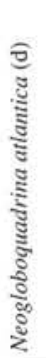 & 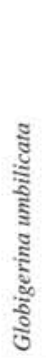 & 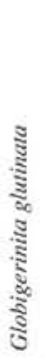 & 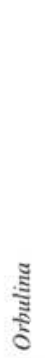 & 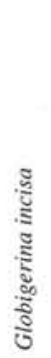 & 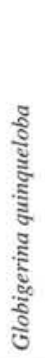 & 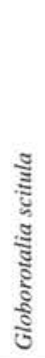 & 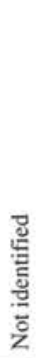 & ฐ్ \\
\hline $\begin{array}{l}16 \mathrm{H}-1,5-10 \\
16 \mathrm{H}-1,85-90 \\
16 \mathrm{H}-2,15-20 \\
16 \mathrm{H}-2,95-100 \\
16 \mathrm{H}-3,25-30 \\
16 \mathrm{H}-3,105-110 \\
16 \mathrm{H}-3,145-150\end{array}$ & $\begin{array}{l}136.1 \\
136.9 \\
137.7 \\
138.5 \\
139.3 \\
140.1 \\
140.5\end{array}$ & $\begin{array}{l}\mathrm{F} \\
\mathrm{F} \\
\mathrm{F} \\
\mathrm{F} \\
\mathrm{F} \\
\mathrm{F} \\
\mathrm{F}\end{array}$ & $\begin{array}{l}2 \\
1 \\
1\end{array}$ & $\begin{array}{r}39 \\
10 \\
5 \\
7 \\
18 \\
1 \\
8\end{array}$ & $\begin{array}{l}197 \\
196 \\
130 \\
209 \\
170 \\
192 \\
197\end{array}$ & $\begin{array}{c}6 \\
24 \\
33 \\
18 \\
38 \\
30 \\
7\end{array}$ & $\begin{array}{l}31 \\
53 \\
66 \\
33 \\
44 \\
33 \\
25\end{array}$ & $\begin{array}{l}28 \\
34 \\
73 \\
31 \\
26 \\
40 \\
80\end{array}$ & $\begin{array}{c}13 \\
6 \\
4 \\
5 \\
9 \\
15 \\
13\end{array}$ & $\begin{array}{l}1 \\
1\end{array}$ & & 1 & $\begin{array}{l}4 \\
3\end{array}$ & $\begin{array}{l}2 \\
1 \\
4 \\
2 \\
1\end{array}$ & $\begin{array}{l}316 \\
327 \\
317 \\
307 \\
310 \\
316 \\
331\end{array}$ \\
\hline
\end{tabular}

Note: See Table 1. 


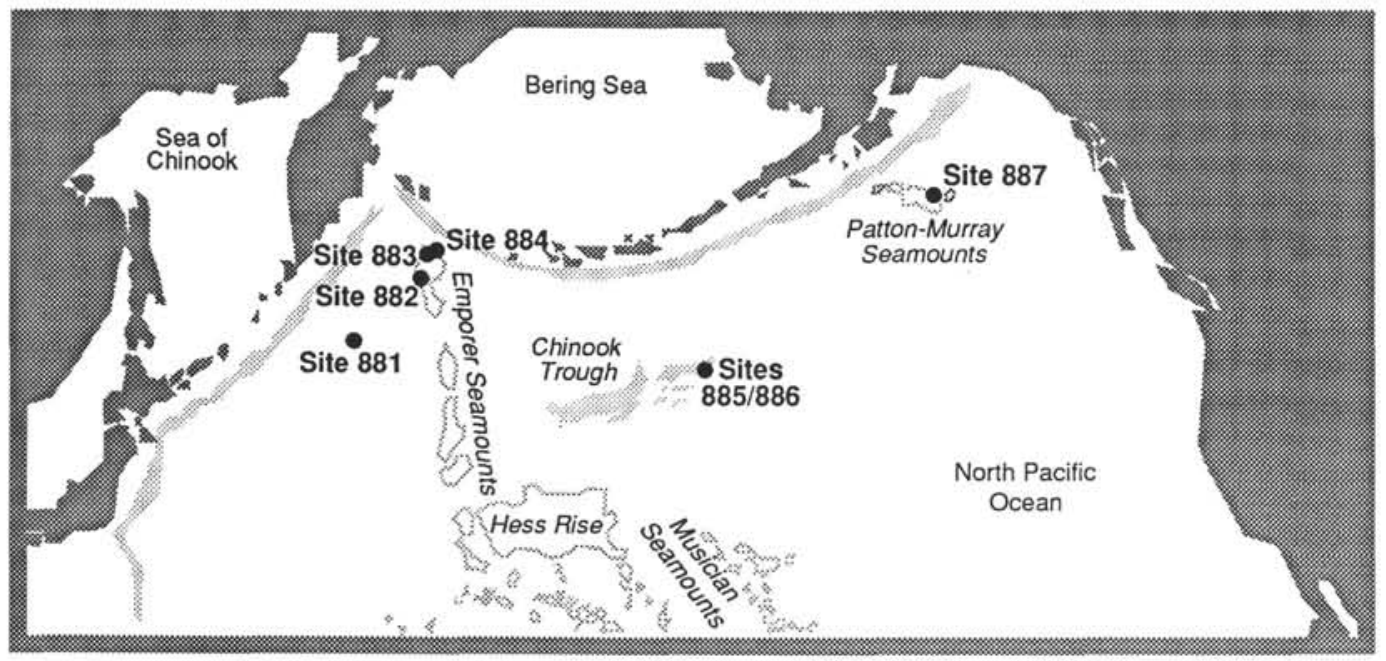

Figure 1. Location of Leg 145 sites in the North Pacific.

This is the first report of Neogloboquadrina atlantica from a high-latitude North Pacific Ocean sequence. We have found that dissolution and overgrowth of surface texture make it difficult to impossible to distinguish $N$. atlantica from Globigerina bulloides under the light microscope. A similar gradation between these taxa was observed by Dowsett and Poore (1990) in the North Atlantic. We estimate that as many as $15 \%$ of individuals identified as either $G$. bulloides or N. atlantica may be incorrectly assigned. For this reason, the counts (Tables 1 and 2) should be considered preliminary until further taxonomic work is performed on the assemblage. Nevertheless, sufficient numbers of unmistakable $N$. atlantica are recognized from the Site 883 material, thus firmly establishing the presence of this taxon at high latitudes throughout the Northern Hemisphere.

Figure 2 indicates two first-order cycles in the planktonic assemblage with broad peaks in the abundance of Globigerina bulloides centered at 128 and $145 \mathrm{~m}$. Higher frequency variability in the planktonic assemblage is superimposed on these two primary cycles. In general, the abundance of cold-water Neogloboquadrina species $(=N$. pachyderma and $N$. atlantica) increases up the sampled interval whereas Globigerina umbilicata decreases up the section.

Pliocene-Pleistocene faunas from DSDP Leg 19, Site 192 (Echols, 1973), from the region immediately to the north of Site 883 , are dominated by $N$. pachyderma with lesser amounts of $G$. bulloides, Globorotalia scitula, and Globigerinita glutinata. Site 883 Pliocene assemblages identified in this paper have approximately the same diversity and represent similar to possibly slightly warmer (because of higher percentages of G. bulloides) conditions than occurred during the late Pleistocene at DSDP Site 192.

\section{Benthic Foraminifers}

A total of 74 samples were analyzed for benthic foraminifers, of which 47 yielded quantitative counts ( $>100$ specimens) (Table 3$)$. We identified 51 species or species groups of calcareous benthic and agglutinated foraminifer taxa. Preservation was moderate to good, with some specimens displaying partial dissolution evident by enlarged pores and absence of the final chamber. The dominant agglutinated taxon, constituting up to $17 \%$ of the fauna, is Eggerella bradyi. Abundant calcareous benthic taxa include Fontbotia wuellerstorfi, Globocassidulina subglobosa, Melonis barleeanum, Pyrgo spp., and Uvigerina proboscidea. Minor components of this fauna are Laticarinina pauperata, Oridorsalis spp., Quinqueloculina spp., Stilostomella lepidula, Ehrenbergina trigona, and Bulimina alazanensis.

The benthic foraminifer fauna from Site 883 is similar to modern lower-middle to upper-lower bathyal (1000-2800 m) faunas from the Pacific (Echols and Armentrout, 1980; Ingle and Keller, 1980; Murray, 1991). Similar faunal associations are related to Deep Oxygen Minimum water in the Pacific (Murray, 1991). This fauna shows little significant variation in species abundances up to approximately $136.5 \mathrm{~m}$, where the abundance of Uvigerina proboscidea increases significantly (up to $44 \%$ abundance) through $133.5 \mathrm{~m}$ (Fig. 3). The association of Uvigerinids with low-oxygen conditions may suggest a significant drop in the oxygen minima at this site. This event is followed by an increase in abundance of Globocassidulina subglobos $a$ and relative decrease in the abundance of the agglutinated taxon Eggerella bradyi (Fig. 3).

\section{SITE 887}

Site 887 is situated on the eastern part of the Patton-Murray Seamount group in the Gulf of Alaska $\left(54^{\circ} 21.921^{\prime} \mathrm{N}, 148^{\circ} 26.765^{\prime} \mathrm{W}\right)$ at a water depth of $3630 \mathrm{~m}$ (Fig. 1). The core site is situated under the center of the Alaskan Gyre and is overlain by Pacific Deep Water. Sea-surface temperatures average $3.5^{\circ} \mathrm{C}$ in February and $12.3^{\circ} \mathrm{C}$ in August (Schweitzer, 1993). High carbonate samples (based upon GRAPE density records) were selected for faunal analysis at this site.

The extraordinary magnetostratigraphic record at this site (Barron et al., this volume) indicates that the high carbonate levels partially coincide with the PRISM time interval. Our uppermost sample corresponds to the middle of the Kaena (C2An.2n), and our lowest sample falls just below the Gauss-Gilbert Chron boundary. Therefore, our samples cover the interval from 3.08 to $3.59 \mathrm{Ma}$ at a sample spacing of approximately 12 k.y. As at Site 883, specimens of Neogloboquadrina pachyderma are predominantly dextrally coiled suggesting correlation to coiling interval CD15 of Lagoe and Thompson, 1988.

\section{Planktonic Foraminifers}

Planktonic foraminifers are common in 36 of the 52 samples examined from Site 887 (Tables 4 and 5). Preservation is consistently better than at Site 883 .

The assemblage at Site 887 is dominated by Globigerina bulloides. Other less common taxa include Neogloboquadrina pachyderma, Globigerina umbilicata, Neogloboquadrina atlantica, Orbulina universa, and Globigerina incisa. Rare occurrences of Globigerinita glutinata, Globorotalia scitula, and Turborotalita quinqueloba complete the assemblage.

Globigerina bulloides is a larger component of the assemblage at Site 887 than at Site 883, suggesting warmer conditions (Fig. 2). Late Pleistocene assemblages from Gulf of Alaska DSDP Leg 18, Sites 179 
Table 3. Occurrences and abundance of benthic foraminifers, Hole 883B.

\begin{tabular}{|c|c|c|c|c|c|c|c|c|c|c|c|c|c|c|c|c|c|c|c|c|c|c|c|c|c|c|c|c|c|}
\hline $\begin{array}{l}\text { Core, section, } \\
\text { interval }(\mathrm{cm})\end{array}$ & $\begin{array}{l}\text { Depth } \\
\text { (mbsf) }\end{array}$ & 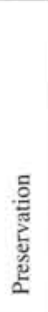 & 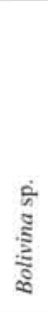 & 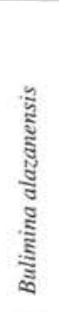 & 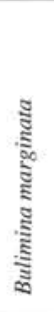 & 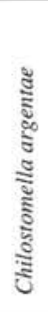 & 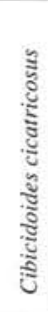 & 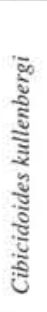 & 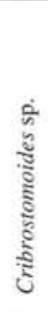 & 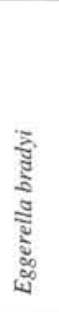 & 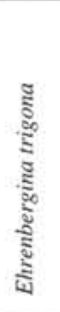 & 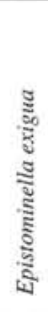 & 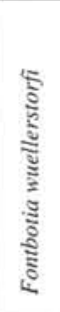 & 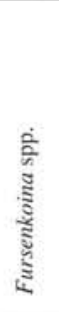 & 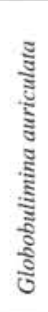 & 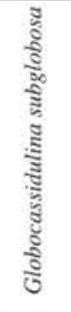 & 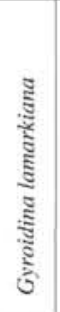 & 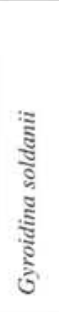 & 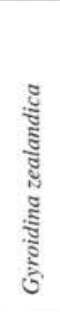 & 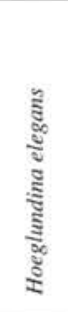 & 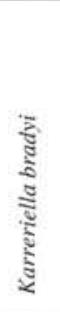 & 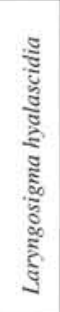 & 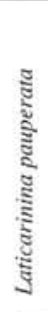 & 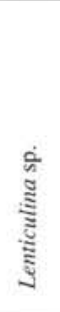 & 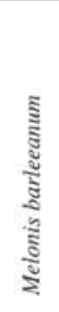 & 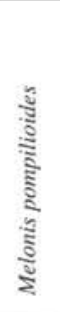 & 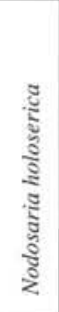 & 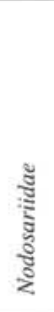 & 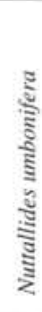 \\
\hline $14 \mathrm{H}-3,102-107$ & 125.92 & $\mathrm{~F}$ & & & & & & & & 11 & & 4 & 21 & & & 18 & & 2 & 4 & & 2 & & & 1 & 36 & & & 8 & \\
\hline $14 \mathrm{H}-3,145-150$ & 126.35 & $\mathrm{~F}$ & & & & & & & & $i$ & & $i$ & 2 & & & 8 & & 6 & 1 & & & & 1 & 1 & 23 & & & 4 & 1 \\
\hline $14 \mathrm{H}-5,45-50$ & 128.35 & $\mathrm{~F}$ & & & & & & & & $\mathrm{i}$ & & & $i$ & & & 3 & 1 & i & & & & & 4 & 1 & 1 & & & 2 & \\
\hline $14 \mathrm{H}-5,85-90$ & 128.75 & $\mathrm{~F}$ & & & & & & & & & & & 1 & & & 1 & & & & & 1 & & 1 & & 2 & & & & \\
\hline $14 \mathrm{H}-6,55-60$ & 129.95 & $\mathrm{~F}$ & & & & 1 & & & & 3 & & & 5 & & & 9 & & 1 & & & & & 2 & 1 & 2 & & & 3 & \\
\hline $14 \mathrm{H}-6,90-95$ & 130.30 & $\mathrm{~F}$ & & & & & & & & 1 & & 1 & 2 & & & 1 & & i & & & 2 & & & & 5 & & & 1 & 1 \\
\hline $14 \mathrm{H}-6,136-14 \mathrm{I}$ & 130.76 & $\mathrm{~F}$ & & & & & & & & & & & 3 & & & 3 & & 2 & & & & & & 1 & 12 & & & 4 & i \\
\hline $14 \mathrm{H}-7,25-30$ & 131.15 & F & & 1 & & & & & & 6 & 2 & & 7 & & & 57 & & $i$ & & & 1 & & 1 & & 13 & & & 2 & i \\
\hline $15 \mathrm{H}-1,45-50$ & 131.45 & $\mathrm{~F}$ & & & & & & 1 & & 10 & & 2 & 35 & & & 2 & 1 & & & & & & 3 & & 8 & & & 4 & \\
\hline $15 \mathrm{H}-2,51-56$ & 133.41 & $\mathrm{~F}$ & & & & 5 & & & & 10 & & & 60 & & & 120 & 4 & 2 & & & & & 3 & & 57 & & & 13 & \\
\hline $15 \mathrm{H}-2,135-140$ & 134.25 & $\mathrm{~F}$ & & & & 2 & & & & 32 & & & 29 & & & 3 & 2 & 3 & I & & 1 & & 4 & I & 27 & & & 4 & \\
\hline $15 \mathrm{H}-3,25-30$ & 134.65 & $\mathrm{~F}$ & & & & 2 & & & & 40 & & & 24 & & & 9 & 8 & 2 & $t$ & & 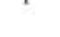 & & 4 & 1 & 44 & & & 9 & \\
\hline $15 \mathrm{H}-3,65-70$ & 135.05 & $\mathrm{~F}$ & & & & 3 & & & & 21 & & & 34 & & & 1 & 4 & $i$ & & & & & 5 & . & 52 & & & 7 & \\
\hline $15 \mathrm{H}-3,102-107$ & 135.42 & $\mathrm{~F}$ & & & 1 & 2 & & & & 38 & & 2 & 31 & & 1 & 3 & & 17 & & & & & 5 & 2 & 47 & & 1 & 4 & \\
\hline $15 \mathrm{H}-3,145-150$ & 135.85 & $\mathrm{~F}$ & & & & 2 & & & & 15 & & 3 & 27 & & & 1 & & 22 & & & 1 & & 1 & 2 & 42 & & . & 4 & \\
\hline $15 \mathrm{H}-4,35-40$ & 136.25 & $\mathrm{~F}$ & & & & 1 & & & & 11 & & 2 & 27 & & & & & 5 & 1 & & 5 & & 4 & & 37 & & & 9 & \\
\hline $15 \mathrm{H}-4,75-80$ & 136.65 & $\mathrm{~F}$ & & & & & & & & 5 & & 4 & 12 & & 4 & & & 3 & & & 3 & & & & 49 & & & 6 & \\
\hline $15 \mathrm{H}-4,111-116$ & 137.01 & $\mathrm{~F}$ & & & & 3 & & & & 17 & & 7 & 16 & & & 2 & 5 & 2 & & & & & & 1 & 30 & & & 3 & \\
\hline $15 \mathrm{H}-5,5-10$ & 137.45 & $\mathrm{~F}$ & & & & 1 & & & & 6 & & 3 & 11 & 1 & & 2 & i & 16 & & 2 & & & 1 & & 29 & & & 8 & \\
\hline $15 \mathrm{H}-5,46-51$ & 137.86 & $\mathrm{~F}$ & & & & & & & & 15 & 2 & & 14 & & & i & & 25 & & & & 1 & & 1 & 34 & & & 5 & \\
\hline $15 \mathrm{H}-5,86-91$ & 138.26 & $\mathrm{~F}$ & & 1 & & & & & & 7 & 3 & & 13 & & & 2 & & 11 & & & & t & 2 & 1 & 19 & & & 7 & \\
\hline $15 \mathrm{H}-5,126-131$ & 138.66 & $\mathrm{~F}$ & & & & & & & & $\mathrm{i}$ & & & ii & & & i & & 19 & & & & & $i$ & 1 & 16 & & & 10 & 1 \\
\hline $15 \mathrm{H}-6,15-20$ & 139.05 & $\mathrm{~F}$ & & 1 & & 2 & & & & 28 & & & 35 & & & 13 & & , & & & & & 3 & 1 & 62 & & & 10 & 1 \\
\hline $15 \mathrm{H}-6,50-55$ & 139.40 & $\mathrm{~F}$ & & i & & 4 & & & & 10 & & & 35 & & & & 22 & 2 & & & & & 5 & 1 & 59 & & & 17 & \\
\hline $15 \mathrm{H}-6,91-96$ & 139.81 & $\mathrm{~F}$ & & & & 2 & & & & 21 & 3 & 5 & 33 & & & 40 & 24 & 2 & & & & & 2 & 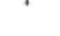 & 69 & & & 8 & \\
\hline $15 \mathrm{H}-7,66-71$ & 140.56 & $\mathrm{~F}$ & & & & $i$ & & & & 17 & & & 25 & & & 25 & & i & & & & & 3 & & 30 & & & 11 & \\
\hline $16 \mathrm{H}-1,5-10$ & 140.95 & $\mathrm{~F}$ & & & & 2 & & & & 7 & & & 14 & & & 17 & 5 & 2 & & & & & 1 & & 15 & & & 4 & \\
\hline $16 \mathrm{H}-1,45-50$ & 141.35 & $\mathrm{~F}$ & & & & & & & & 7 & & & 12 & & & 7 & 8 & & & & & & & & 15 & & & 4 & \\
\hline $16 \mathrm{H}-1,85-90$ & 141.75 & $\mathrm{~F}$ & & & 1 & 3 & & & & 12 & & & 33 & & & 28 & 3 & & & & & & 4 & 1 & 57 & & & 7 & \\
\hline $16 \mathrm{H}-1,125-130$ & 142.15 & $\mathrm{~F}$ & & & & 2 & & & & 11 & & 1 & 26 & & & 15 & & 1 & & & & & 2 & 1 & 36 & & & 8 & \\
\hline $16 \mathrm{H}-2,15-20$ & 142.55 & $\mathrm{~F}$ & & & & 2 & & & & 11 & & & 32 & & & 8 & 2 & & & & & & & & 44 & & & 4 & \\
\hline $16 \mathrm{H}-2,50-52$ & 142.90 & $\mathrm{~F}$ & & & 1 & 8 & & & & 24 & 1 & 1 & 36 & & & 4 & 8 & & & & & & 6 & & 37 & & & 4 & \\
\hline $16 \mathrm{H}-2,135-140$ & 143.75 & $\mathrm{~F}$ & 1 & & & & & & & & 17 & $\mathrm{i}$ & 13 & & & & 3 & & & & & & 2 & & 12 & & & 7 & \\
\hline $16 \mathrm{H}-3,25-30$ & 144.15 & $\mathrm{~F}$ & & & & & & & & 20 & & & 19 & & & & 4 & & & & & & & & 15 & & & 4 & \\
\hline $16 \mathrm{H}-3,65-70$ & 144.55 & $\mathrm{~F}$ & & & & & & & 1 & 15 & 2 & 2 & 36 & & & & 3 & & & & & & 3 & 1 & 36 & & & 14 & \\
\hline $16 \mathrm{H}-3,101-106$ & 144.91 & $\mathrm{~F}$ & & & & 2 & & & & 16 & & & 21 & & & & 6 & & & & & & i & & 16 & & & 12 & \\
\hline $16 \mathrm{H}-3,145-150$ & 145.35 & $\mathrm{~F}$ & & & & 2 & & 4 & & & 1 & & 26 & & & 1 & 8 & & & & & & & & 14 & & & 13 & \\
\hline $16 \mathrm{H}-4,35-40$ & 145.75 & $\mathrm{~F}$ & & & & 3 & & & & 14 & & 1 & 32 & & & 1 & $\begin{array}{l}\circ \\
4\end{array}$ & 2 & & & & & 1 & 6 & 13 & & & 9 & \\
\hline $16 \mathrm{H}-4,111-116$ & 146.51 & $\mathrm{~F}$ & & & & 2 & & & & 12 & & 2 & 20 & & & & 11 & 2 & & & & & & 2 & 12 & & & 11 & \\
\hline $16 \mathrm{H}-5,5-10$ & 146.95 & $\mathrm{~F}$ & & & & & & & & 14 & 1 & $i$ & 46 & & & & 5 & 1 & & & & & 3 & 6 & 26 & & & 19 & \\
\hline $16 \mathrm{H}-5,45-50$ & 147.35 & $\mathrm{~F}$ & & & & 5 & & & & 15 & & & 56 & & & & 13 & 2 & & & & & 4 & 5 & 23 & & & 54 & \\
\hline $16 \mathrm{H}-5,85-90$ & 147.75 & $\mathrm{~F}$ & & & & & & & & 4 & & & 23 & & & & 7 & 3 & & & & & 1 & 1 & 10 & & & 16 & \\
\hline $16 \mathrm{H}-5,120-125$ & 148.10 & $\mathrm{~F}$ & & & & 2 & & & & 6 & & & 27 & & & 1 & & 6 & & & & & & 1 & 30 & & & 20 & \\
\hline $16 \mathrm{H}-6,15-20$ & 148.55 & $\mathrm{~F}$ & & & & 2 & & & & 1 & & & 22 & & & 2 & 4 & 5 & & & & & & & 16 & & & 2 & \\
\hline $16 \mathrm{H}-6,50-55$ & 148.90 & $\mathrm{~F}$ & & & & & & & & 11 & & 3 & 9 & & & 9 & 15 & 3 & & & & & & & 9 & 5 & & 9 & \\
\hline $16 \mathrm{H}-6,135-140$ & 149.75 & $\mathrm{~F}$ & & & & & & 2 & & 3 & & 1 & 7 & & & & & 12 & & & & & & & 20 & & & 2 & \\
\hline $16 \mathrm{H}-7,25-30$ & 150.15 & $\mathrm{~F}$ & & & & & 3 & & & 2 & & 3 & 9 & & & & & 24 & & & & & & 1 & 22 & & & $i$ & \\
\hline
\end{tabular}


Table 3 (continued).

\begin{tabular}{|c|c|c|c|c|c|c|c|c|c|c|c|c|c|c|c|c|c|c|c|c|c|c|c|c|c|c|c|c|}
\hline $\begin{array}{l}\text { Core, section, } \\
\text { interval (cm) }\end{array}$ & $\begin{array}{l}\text { Depth } \\
\text { (mbsf) }\end{array}$ & 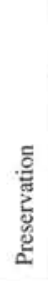 & 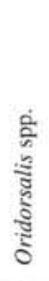 & 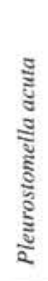 & 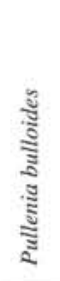 & 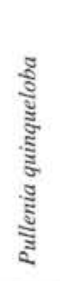 & 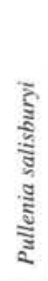 & $\begin{array}{l}0 \\
\hat{0} \\
0 \\
0 \\
\vdots \\
2\end{array}$ & 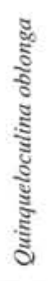 & 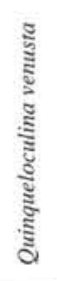 & 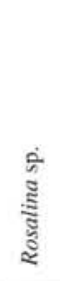 & 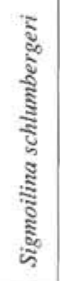 & 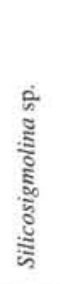 & 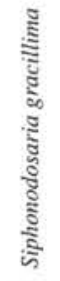 & 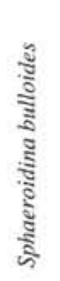 & 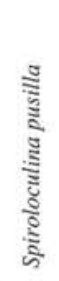 & 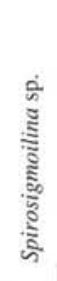 & 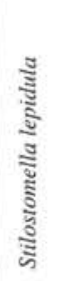 & 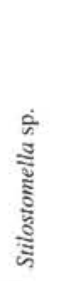 & 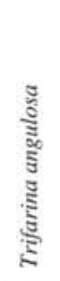 & 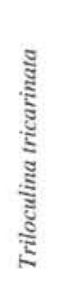 & 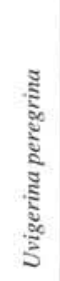 & 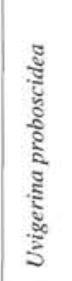 & 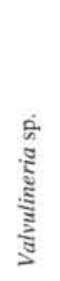 & 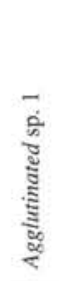 & 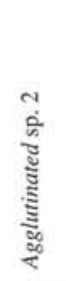 & 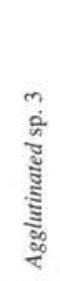 & Total \\
\hline $14 \mathrm{H}-3,102-107$ & 125.92 & $\mathrm{~F}$ & 1 & & & & 2 & 16 & & & & & & & & & & & & & 2 & & 16 & & & & & 144 \\
\hline $14 \mathrm{H}-3,145-150$ & 126.35 & $\mathrm{~F}$ & & & & & 2 & 12 & & & & & & & & & & & & & & 1 & 5 & & & & & 68 \\
\hline $14 \mathrm{H}-5,45-50$ & 128.35 & $\mathrm{~F}$ & 2 & & & & $i$ & 1 & & & & & & & & & 1 & & & & & & & & & & & 20 \\
\hline $14 \mathrm{H}-5,85-90$ & 128.75 & $\mathrm{~F}$ & & & & & i & 1 & & & & & & & & & & & & & & & & & & & 1 & 9 \\
\hline $14 \mathrm{H}-6,55-60$ & 129.95 & $\mathrm{~F}$ & 3 & & & 1 & & 2 & & & & & & & & & & 1 & & & & & & & & & i & 35 \\
\hline $14 \mathrm{H}-6,90-95$ & 130.30 & $\mathrm{~F}$ & 3 & & & 1 & & 11 & & 3 & & & & & & & & I & & & 1 & & & & & & & 33 \\
\hline $14 \mathrm{H}-6,136-141$ & 130.76 & $\mathrm{~F}$ & 1 & & & 2 & & 4 & & 3 & & & & & & & & & & & 1 & & & & & & & 33 \\
\hline $14 \mathrm{H}-7,25-30$ & 131.15 & $\mathrm{~F}$ & 2 & & 2 & 4 & & 5 & & & & & & & 1 & & & 1 & & & & & 2 & & & & & 109 \\
\hline $15 \mathrm{H}-1,45-50$ & 131.45 & $\mathrm{~F}$ & 2 & 1 & 2 & $\frac{4}{3}$ & & 5 & & & & & & & 1 & & & 1 & 1 & & & & 13 & 1 & & & & 92 \\
\hline $15 \mathrm{H}-2,51-56$ & 133.41 & $\mathrm{~F}$ & 20 & & 3 & 9 & & 40 & & 5 & & & & & & & & 1 & i & & & & 45 & & & & & 398 \\
\hline $15 \mathrm{H}-2,135-140$ & 134.25 & $\mathrm{~F}$ & 3 & & 2 & 8 & & $\begin{array}{r}40 \\
9\end{array}$ & & & & & & & & & 1 & 9 & & & 3 & & 53 & 2 & & & & 199 \\
\hline $15 \mathrm{H}-3,25-30$ & 134.65 & $\mathrm{~F}$ & 6 & 1 & 1 & 9 & 1 & 19 & & 7 & & & & & & & 1 & 3 & & & & & 69 & & & & 1 & 261 \\
\hline $15 \mathrm{H}-3,65-70$ & 135.05 & $\mathrm{~F}$ & 2 & 2 & & 2 & & 6 & & & & & & 1 & & & & & & & & & 111 & & & & 2 & 254 \\
\hline $15 \mathrm{H}-3,102-107$ & 135.42 & $\mathrm{~F}$ & & & 2 & 3 & & 24 & & 2 & & 1 & & i & & & & & & & & & 112 & 1 & & & & 300 \\
\hline $15 \mathrm{H}-3,145-150$ & 135.85 & $\mathrm{~F}$ & 1 & & & & & 41 & 4 & & & & & & & & & & & & & & 43 & & & & & 207 \\
\hline $15 \mathrm{H}-4,35-40$ & 136.25 & $\mathrm{~F}$ & & & & 2 & & 19 & 2 & & & & & 1 & & & & & & & & & 50 & & & & 1 & 177 \\
\hline $15 \mathrm{H}-4,75-80$ & 136.65 & $\mathrm{~F}$ & & & 2 & 2 & & 14 & & & & & & & & & & 1 & & & & & 26 & & & & & 131 \\
\hline $15 \mathrm{H}-4,111-116$ & 137.01 & $\mathrm{~F}$ & 2 & 1 & i & & 3 & 34 & & & & & & & 1 & & & & & & & & 12 & 3 & & & & 143 \\
\hline $15 \mathrm{H}-5,5-10$ & 137.45 & $\mathrm{~F}$ & 5 & & 8 & 2 & 2 & 4 & & & & & 1 & & & & & & & & & & 2 & i & & & 1 & 107 \\
\hline $15 \mathrm{H}-5,46-5 \mathrm{I}$ & 137.86 & $\mathrm{~F}$ & 11 & 1 & 10 & & 6 & 3 & & & & & i & & & & & 3 & & & & & 10 & & & & i & 144 \\
\hline $15 \mathrm{H}-5,86-91$ & 138.26 & $\mathrm{~F}$ & 1 & $\mathrm{i}$ & 2 & & 2 & 13 & & 1 & & & & & & & & 2 & & & 3 & & 6 & & & & . & 96 \\
\hline $15 \mathrm{H}-5,126-131$ & 138.66 & $\mathrm{~F}$ & 10 & & 6 & & 2 & 19 & & 2 & & & & & 2 & & & & & & $\overrightarrow{1}$ & & 22 & & & & 2 & 127 \\
\hline $15 \mathrm{H}-6,15-20$ & $\begin{array}{l}130.00 \\
139.05\end{array}$ & $\mathrm{~F}$ & 22 & & 10 & & 2 & 23 & & 1 & & & & & 2 & & & 1 & & & 1 & & 15 & & I & & 2 & 230 \\
\hline $15 \mathrm{H}-6,50-55$ & 139.40 & $\mathrm{~F}$ & 14 & & & & 2 & 22 & & 16 & & & & & 1 & & & 1 & & 2 & 3 & & 3 & 3 & 1 & & & 222 \\
\hline $15 \mathrm{H}-6,91-96$ & 139.81 & $\mathrm{~F}$ & 22 & & 1 & & 2 & 24 & & 3 & & & & & & & & 2 & & 2 & & & 7 & $i$ & & 1 & & 272 \\
\hline $15 \mathrm{H}-7,66-71$ & 140.56 & F & 8 & & & & 4 & 21 & & & & & & & & & & & & & & & 5 & & & 1 & & 152 \\
\hline $16 \mathrm{H}-1,5-10$ & 140.95 & $\mathrm{~F}$ & 10 & & 3 & & & 12 & & 3 & & & & & 1 & & & 2 & & & & & 6 & & & & & 104 \\
\hline $16 \mathrm{H}-1,45-50$ & 141.35 & $\mathrm{~F}$ & 8 & & & & 1 & 18 & & 3 & & & & & & & & & & & & & & & & & & 83 \\
\hline $16 \mathrm{H}-1,85-90$ & 141.75 & $\mathrm{~F}$ & 15 & & & & & 18 & & & & & & & 2 & & & 1 & & & & & 3 & 7 & & 2 & & 197 \\
\hline $16 \mathrm{H}-1,125-130$ & 142.15 & $\mathrm{~F}$ & 11 & & & & 2 & 17 & & 3 & & & & & $i$ & 1 & & 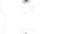 & & & 2 & & 2 & & & & & 142 \\
\hline $16 \mathrm{H}-2,15-20$ & 142.55 & $\mathrm{~F}$ & 18 & & 7 & & 2 & 7 & & & & & & & . & & & 1 & & & 3 & & 3 & 1 & & & & 145 \\
\hline $16 \mathrm{H}-2,50-52$ & 142.90 & $\mathrm{~F}$ & 19 & & 15 & & i & 14 & 1 & & & & & & 2 & 1 & & & & & i & & & 7 & & 1 & & 192 \\
\hline $16 \mathrm{H}-2,135-140$ & 143.75 & $\mathrm{~F}$ & 15 & & 7 & & 7 & 31 & $\mathrm{i}$ & & & & & & 2 & & & & & & & & & & & 1 & & 120 \\
\hline $16 \mathrm{H}-3,25-30$ & 144.15 & $\mathrm{~F}$ & 5 & & 2 & & 1 & 41 & i & & & & & & & & & & & & & & & & & 8 & & 120 \\
\hline $16 \mathrm{H}-3,65-70$ & 144.55 & $\mathrm{~F}$ & 14 & & 6 & & 4 & 29 & & 9 & & & & & & & & & & & & & 6 & & & $\begin{array}{l}\circ \\
4\end{array}$ & & 185 \\
\hline $16 \mathrm{H}-3,10:-106$ & 144.91 & $\mathrm{~F}$ & 7 & 1 & 3 & 2 & 2 & 27 & & & & & & & 2 & & & & & & 4 & & 0 & & & 1 & & 123 \\
\hline $16 \mathrm{H}-3,145-150$ & 145.35 & $\mathrm{~F}$ & 11 & 1 & 7 & 2 & & 16 & & & & & & & 3 & & & & & & 4 & & 20 & & & $\begin{array}{l}1 \\
3\end{array}$ & & 138 \\
\hline $16 \mathrm{H}-4,35-40$ & 145.75 & $\mathrm{~F}$ & 4 & & 11 & 2 & & 25 & & & & & & & & & & & & 1 & 8 & & 26 & & & & & 162 \\
\hline $16 \mathrm{H}-4,111-116$ & 146.51 & $\mathrm{~F}$ & 7 & & 12 & & & 42 & & 9 & & & & & & & & & & & & & 1 & & 4 & 4 & & 151 \\
\hline $16 \mathrm{H}-5,5-10$ & 146.95 & $\mathrm{~F}$ & 5 & & 10 & 4 & & 34 & & 1 & & & & & & & & 1 & & 1 & 2 & & 12 & 1 & 6 & 4 & & 203 \\
\hline $16 \mathrm{H}-5,45-50$ & 147.35 & $\mathrm{~F}$ & 5 & & 9 & 1 & 7 & 49 & & & & & & & & 1 & & i & & 1 & 9 & & 13 & & 14 & 16 & & 303 \\
\hline $16 \mathrm{H}-5,85-90$ & 147.75 & $\mathrm{~F}$ & 2 & & 8 & i & & 52 & & & & & & & 2 & & & 2 & & & 4 & & 4 & & & & & 144 \\
\hline $16 \mathrm{H}-5,120-125$ & 148.10 & F & 3 & 1 & 10 & & 3 & 70 & & & & & & & & & & 1 & & & 10 & & & & & & & 191 \\
\hline $16 \mathrm{H}-6,15-20$ & 148.55 & $\mathrm{~F}$ & 1 & & 3 & 1 & 1 & 35 & & & & & & & & & & & & & & & & & & & & 95 \\
\hline $16 \mathrm{H}-6,50-55$ & 148.90 & $\mathrm{~F}$ & 2 & 1 & 14 & & 4 & 25 & & & 2 & & & & & & & & & & 1 & & & 2 & & & & 124 \\
\hline $16 \mathrm{H}-6,135-140$ & 149.75 & $\mathrm{~F}$ & & & & & 1 & 6 & & & 2 & & & & 2 & & & & & & & & & & & & & 58 \\
\hline $16 \mathrm{H}-7,25-30$ & 150.15 & $\mathrm{~F}$ & 1 & & & & & 12 & & & & & & & 2 & & & & & & 1 & & & & & & & 81 \\
\hline
\end{tabular}

Notes: See Table 1. Abundance $=$ number of specimens 
Table 4. Occurrences of planktonic foraminifers, Hole 887A.

\begin{tabular}{|c|c|c|c|c|c|c|c|c|c|c|c|c|c|c|c|}
\hline $\begin{array}{l}\text { Core, section, } \\
\text { interval (cm) }\end{array}$ & $\begin{array}{l}\text { Depth } \\
\text { (mbsf) }\end{array}$ & 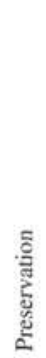 & 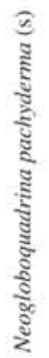 & 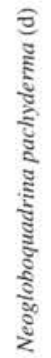 & 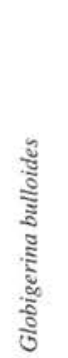 & 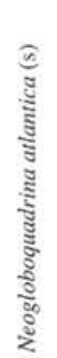 & 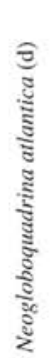 & 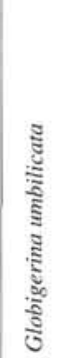 & 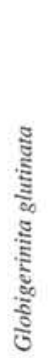 & 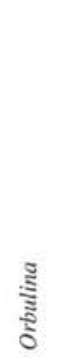 & 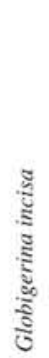 & 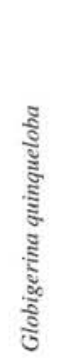 & 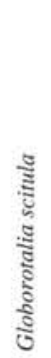 & 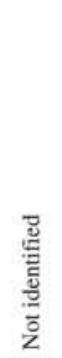 & 胥 \\
\hline $11 \mathrm{H}-7,18-20$ & 101.38 & $\mathrm{~F}$ & 1 & 4 & 31 & 11 & 20 & 24 & 3 & & & 1 & & & 95 \\
\hline $11 \mathrm{H}-7,41-43$ & 101.61 & G & & & 2 & & 4 & 2 & & & & & 1 & & 9 \\
\hline $12 \mathrm{H}-1,26-28$ & 101.96 & G & 1 & 5 & $23 i$ & 12 & 35 & 26 & 1 & 1 & & & & 5 & 317 \\
\hline $12 \mathrm{H}-1,43-45$ & 102.13 & G & & 6 & 250 & 9 & 25 & 4 & 2 & & 5 & & 1 & 3 & 305 \\
\hline $12 \mathrm{H}-1,66-68$ & 102.36 & $\mathrm{~F}$ & & 2 & 91 & 30 & 35 & 10 & & & & & & 1 & 169 \\
\hline $12 \mathrm{H}-\mathrm{I} .83-85$ & 102.53 & $\mathrm{~F}$ & & 4 & 11 & 5 & 12 & & & & & & & & 32 \\
\hline $12 \mathrm{H}-1,105-107$ & 102.75 & $\mathrm{~F}$ & & 5 & 2 & 2 & 4 & & 1 & & & & & & 14 \\
\hline $12 \mathrm{H}-1.127-129$ & 102.97 & $\mathrm{~F}$ & 2 & 2 & 240 & 13 & 14 & 29 & & & 6 & & & 7 & 313 \\
\hline $12 \mathrm{H}-1,145-147$ & 103.15 & $\mathrm{~F}$ & & & 222 & 12 & 7 & $i$ & & 4 & & & 1 & & 247 \\
\hline $12 \mathrm{H}-2,16-18$ & 103.36 & G & & 2 & 119 & 42 & 67 & 10 & & & & & & 1 & 241 \\
\hline $12 \mathrm{H}-2,37-39$ & 103.57 & G & & & & 1 & 1 & & & & & & & & 2 \\
\hline $12 \mathrm{H}-2,55-57$ & 103.75 & $\mathrm{~F}$ & & & 2 & & & & & & & & & & 2 \\
\hline $12 \mathrm{H}-2,73-75$ & 103.93 & $\mathrm{~F}$ & 1 & 7 & 128 & 23 & 26 & 20 & 1 & & & & 3 & 18 & 227 \\
\hline $12 \mathrm{H}-2,95-97$ & 104.15 & $\mathrm{~F}$ & i & 2 & 187 & 47 & 42 & 19 & & & & & & 1 & 299 \\
\hline $12 \mathrm{H}-2,113-115$ & 104.33 & G & 5 & & 207 & 5 & 8 & 25 & & & & & 3 & 7 & 260 \\
\hline $12 \mathrm{H}-2,135-137$ & 104.55 & G & 1 & 3 & 178 & 26 & 21 & 22 & & & 4 & & 1 & 2 & 258 \\
\hline $12 \mathrm{H}-3,5-7$ & 104.75 & $\mathrm{~F}$ & & & 125 & 13 & 9 & & & & & & & 6 & 153 \\
\hline $12 \mathrm{H}-3,23-25$ & 104.93 & $\mathrm{~F}$ & & 2 & 204 & 43 & 38 & 23 & 2 & & & & 1 & & 313 \\
\hline $12 \mathrm{H}-3,63-65$ & 105.33 & $\mathrm{~F}$ & 2 & 2 & 210 & 53 & 42 & 14 & 1 & & 2 & & & & 326 \\
\hline $12 \mathrm{H}-3,85-87$ & 105.55 & $\mathrm{~F}$ & & $i$ & 243 & 41 & 20 & 18 & & & & & & 1 & 324 \\
\hline $12 \mathrm{H}-3,105-107$ & 105.75 & $\mathrm{~F}$ & & 1 & 186 & 72 & 41 & 15 & & & & & & 1 & 316 \\
\hline $12 \mathrm{H}-3,127-129$ & 105.97 & $\mathrm{~F}$ & 3 & & 185 & 24 & 8 & 62 & 27 & & & & & 7 & 316 \\
\hline $12 \mathrm{H}-3,145-147$ & 106.15 & $\mathrm{~F}$ & & & 22 & 1 & i & 12 & 1 & & & & 1 & & 38 \\
\hline $12 \mathrm{H}-4,13-15$ & 106.33 & $\mathrm{~F}$ & & & 24 & & & 23 & & & & & & & 47 \\
\hline $12 \mathrm{H}-4,33-35$ & 106.53 & $\mathrm{~F}$ & & & 7 & & & & 1 & & & & & & 8 \\
\hline $12 \mathrm{H}-4.77-79$ & 106.97 & $\mathrm{~F}$ & & & 91 & 6 & 4 & 16 & & & & & & 4 & 121 \\
\hline $12 \mathrm{H}-4,95-97$ & 107.15 & F & 9 & 2 & 188 & 31 & 17 & 45 & & & 2 & & & 6 & 300 \\
\hline $12 \mathrm{H}-4,113-115$ & 107.33 & $\mathrm{~F}$ & & & 24 & 1 & 1 & 3 & & & & & & & 29 \\
\hline $12 \mathrm{H}-4,135-137$ & 107.55 & G & & & 2 & & & & & & & & & & 2 \\
\hline $12 \mathrm{H}-5,5-7$ & 107.75 & G & & 2 & 221 & 32 & 23 & 43 & 1 & & & & & 2 & 324 \\
\hline $12 \mathrm{H}-5,23-25$ & 107.93 & G & & & 114 & 19 & 22 & 40 & $i$ & & 1 & & & & 197 \\
\hline $12 \mathrm{H}-5,43-45$ & 108.13 & $\mathrm{~F}$ & 4 & 3 & 196 & 41 & 26 & 48 & & 2 & 2 & & & 1 & 323 \\
\hline $12 \mathrm{H}-5,66-68$ & 108.36 & $\mathrm{~F}$ & 3 & & 161 & 24 & 11 & 103 & & & $i$ & & & 3 & 306 \\
\hline $12 \mathrm{H}-5,83-85$ & 108.53 & G & & & 87 & 4 & 1 & 45 & & & & & & & 137 \\
\hline $12 \mathrm{H}-5,105-107$ & 108.75 & $\mathrm{~F}$ & 1 & 1 & 262 & 11 & 12 & 23 & & 43 & & & & & 353 \\
\hline $12 \mathrm{H}-5,145-147$ & 109.15 & $\mathrm{~F}$ & & & 4 & & 1 & & & 4 & & & & & 9 \\
\hline $12 \mathrm{H}-6,13-15$ & 109.33 & $\mathrm{~F}$ & & & 214 & 1 & 8 & 17 & & 30 & & & & & 270 \\
\hline $12 \mathrm{H}-6,34-36$ & 109.54 & $\mathrm{~F}$ & 2 & 2 & 192 & 24 & 28 & 24 & & 66 & 2 & & 3 & 4 & 347 \\
\hline $12 \mathrm{H}-6,95-97$ & 110.15 & G & & & 49 & 2 & 3 & 11 & & 9 & & & & & 74 \\
\hline $12 \mathrm{H}-7,5-7$ & 110.75 & $\mathrm{~F}$ & & & 182 & 38 & 22 & 79 & & 5 & 2 & & 2 & 5 & 335 \\
\hline $12 \mathrm{H}-7,66-68$ & 111.36 & G & & & 1 & 3 & 1 & & 1 & & & & 1 & & 7 \\
\hline
\end{tabular}

Note: See Table 1.

Table 5. Occurrences of planktonic foraminifers, Hole 887C.

\begin{tabular}{|c|c|c|c|c|c|c|c|c|c|c|c|c|c|}
\hline $\begin{array}{l}\text { Core, section, } \\
\text { interval }(\mathrm{cm})\end{array}$ & $\begin{array}{l}\text { Depth } \\
\text { (mbsf) }\end{array}$ & 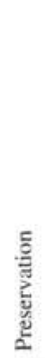 & 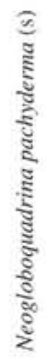 & 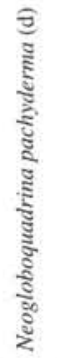 & 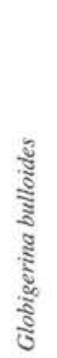 & 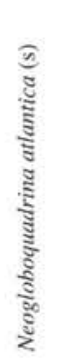 & 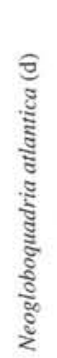 & 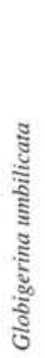 & 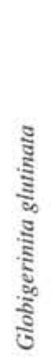 & $\frac{\bar{\Xi}}{\bar{z}}$ & 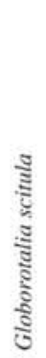 & 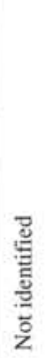 & Е్ \\
\hline $12 \mathrm{H}-1,45-47$ & 93.75 & G & 6 & 5 & 262 & 19 & 10 & 13 & & & & 1 & 316 \\
\hline $12 \mathrm{H}-1,65-67$ & 93.95 & $\mathrm{G}$ & & & & 2 & & 1 & & & & & 3 \\
\hline $12 \mathrm{H}-1,125-127$ & 94.55 & $\mathrm{~F}$ & & 11 & 195 & 29 & 52 & 3 & & & & 1 & 291 \\
\hline $12 \mathrm{H}-\mathrm{l}, 145-147$ & 94.75 & $\mathrm{~F}$ & 5 & 38 & 143 & 22 & 115 & 14 & & & 1 & 2 & 340 \\
\hline $13 \mathrm{H}-1,5-7$ & 102.85 & G & & & 170 & 27 & 19 & 2 & & & 2 & 0 & 220 \\
\hline $13 \mathrm{H}-1,25-27$ & 103.05 & G & 3 & & 45 & 29 & 29 & 29 & 1 & & & 3 & 139 \\
\hline $13 \mathrm{H}-1,45-47$ & 103.25 & $\mathrm{~F}$ & & & 3 & 8 & 6 & 9 & & & & & 26 \\
\hline $13 \mathrm{H}-1,65-67$ & 103.45 & $\mathrm{~F}$ & & & 18 & 10 & 8 & 22 & & 1 & & & 59 \\
\hline $13 \mathrm{H}-1,85-87$ & 103.65 & $\mathrm{~F}$ & 5 & 1 & 127 & 40 & 25 & 23 & & & 1 & 7 & 229 \\
\hline $13 \mathrm{H}-1,105-107$ & 103.85 & $\mathrm{~F}$ & 2 & 2 & 110 & 8 & 6 & 16 & & & & 2 & 146 \\
\hline $13 \mathrm{H}-1,145-147$ & 104.25 & $\mathrm{~F}$ & & & 118 & 19 & 4 & 45 & & & & 3 & 189 \\
\hline
\end{tabular}

Note: See Table 1. 

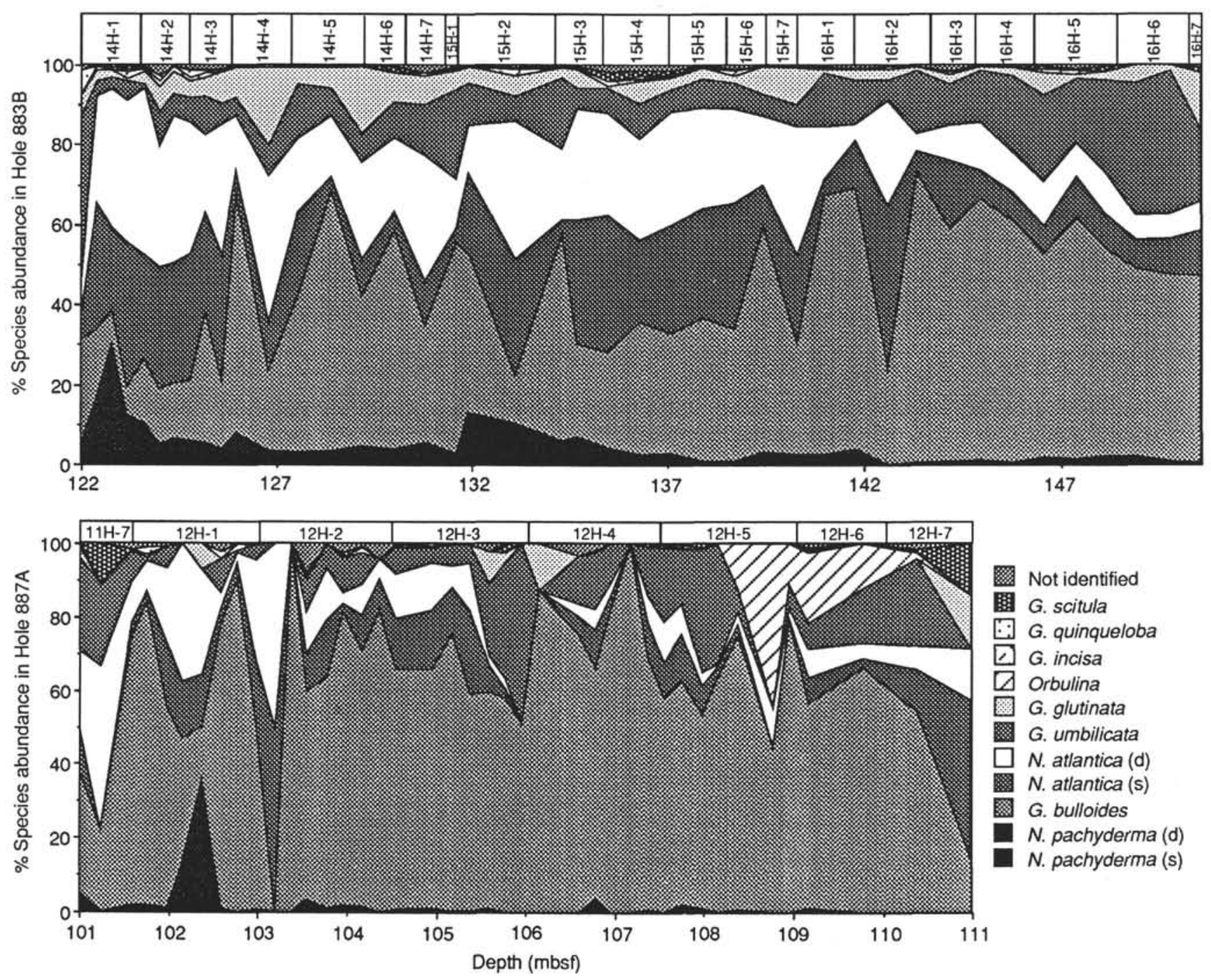

Figure 2. Planktonic foraminifer abundances at Sites 883 and 887.

and 180 (Ingle, 1973) are similar to Pleistocene assemblages in the western North Pacific and include predominantly N. pachyderma, with lesser amounts of $G$. bulloides and rare occurrences of $G$. umbilicata, Globigerinita glutinata, and Turborotalita quinqueloba. The Pliocene assemblage recovered from Site 883 has significantly greater numbers of Globigerina bulloides and indicates a warmer water assemblage. Near the base of Core 145-887A-12H,Orbulina universa is present for a short interval at abundances of just over $10 \%$. Thus, the Pliocene assemblage at Site 887 represents warmer conditions than the Pleistocene assemblages of this region or western subarctic North Pacific.

\section{Benthic Foraminifers}

Benthic foraminifers were recovered from 46 samples from Holes $887 \mathrm{~A}$ ( 31 samples) and $887 \mathrm{C}$ ( 15 samples) (Tables 6 and 7; Fig. 4). The fauna is dominated by calcareous benthic taxa with one prominent agglutinated taxon, Eggerella bradyi. Abundant calcareous benthic taxa include Nuttallides umbonifera, Globocassidulina subglobosa, Oridorsalis spp., Melonis barleeanum, Pullenia bulloides and Epistominella exigua (Tables 6 and 7). Minor components of this fauna are Bolivina decussata, Cibicidoides kullenbergi, Fontbotia wuellerstorfi, Gyroidina spp., Laticarinata pauperata, Sphaeroidina bulloides, Trifarina angulosa, and Uvigerina proboscidea. Preservation of the fauna is moderate to good, with some specimens displaying dissolution features such as enlarged pores and broken or missing final chambers.

The benthic foraminifer fauna recovered from Site 887 is typical of a Neogene Pacific Ocean abyssal benthic foraminifer fauna. The dominance of Nuttallides umbonifera associated with other deep-water benthic foraminifers such as Oridorsalis spp. and Epistominella exigua is similar to the deep-water Pliocene-Pleistocene assemblage described from the Gulf of Alaska, DSDP Site 178 (Ingle, 1973) and equatorial Pacific deep-sea benthic distributions described by Thomas (1985) and Ingle and Keller (1980). This fauna bears resemblance to the Nuttallides umboniferus association in the Pacific (Murray, 1991) that is related to Antarctic Bottom Water (AABW) near the lysocline-carbonate compensation depth (CCD) boundary. This fauna shows little variation throughout the section studied. However, an increase in the abundance of Epistominella exigua is observed from 102.97 through $101.75 \mathrm{~m}$ of the core. In addition, three distinct intervals (111.36-111.14 m, 110.33 $\mathrm{m}$, and 109.93-109.75 m) have very low abundances or are barren of calcareous benthic foraminifers, indicating possible dissolution events.

\section{DISCUSSION}

\section{Neogloboquadrina atlantica (Berggren)}

Poore and Berggren (1975) reexamined the gross morphology and test surface ultrastructure of Neogloboquadrina atlantica (Berggren). 
Table 6. Occurrences and abundance of benthic foraminifers, Hole 887A.

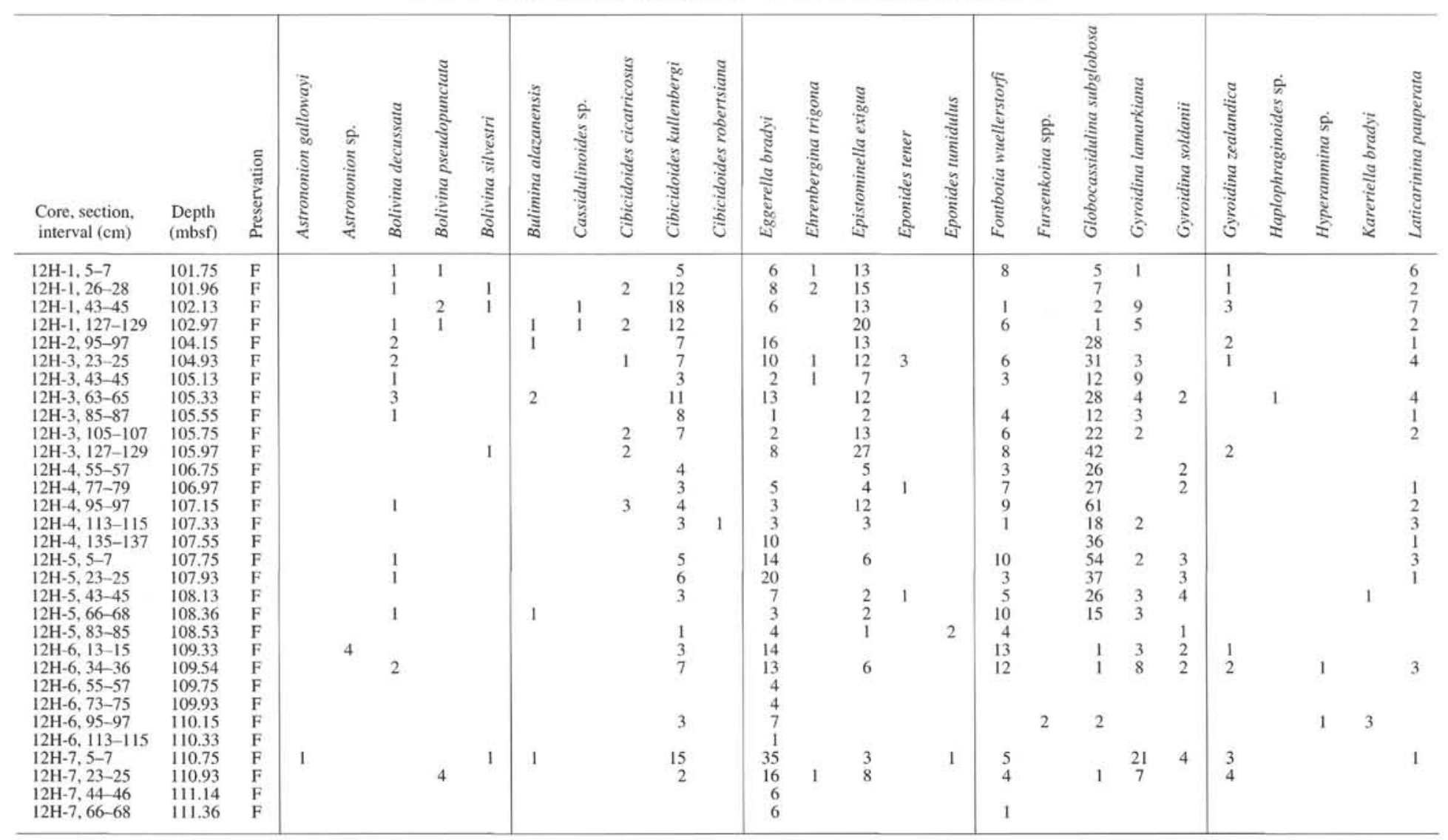


Table 6 (continued).

\begin{tabular}{|c|c|c|c|c|c|c|c|c|c|c|c|c|c|c|c|c|c|c|c|c|c|c|c|c|c|c|c|}
\hline $\begin{array}{l}\text { Core, section, } \\
\text { interval }(\mathrm{cm})\end{array}$ & $\begin{array}{l}\text { Depth } \\
\text { (mbsf) }\end{array}$ & 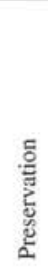 & 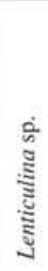 & 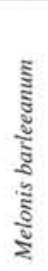 & 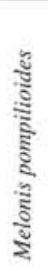 & 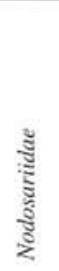 & 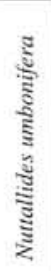 & 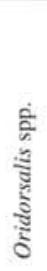 & 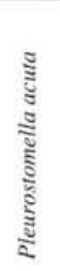 & 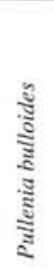 & 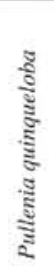 & 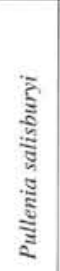 & 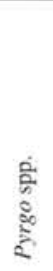 & 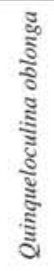 & 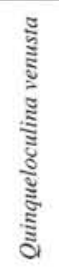 & 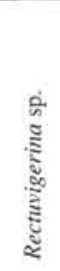 & 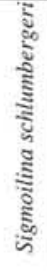 & 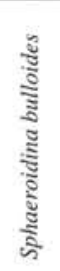 & 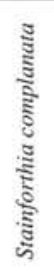 & 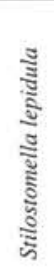 & 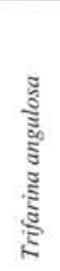 & 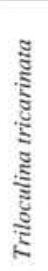 & 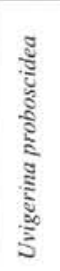 & 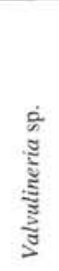 & 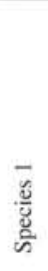 & 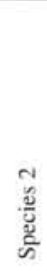 & Total \\
\hline $12 \mathrm{H}-1,5-7$ & 101.75 & $\mathrm{~F}$ & & 28 & & & 42 & 41 & & 23 & & & 4 & & & 3 & & & & 1 & & & 2 & & & & 192 \\
\hline $12 \mathrm{H}-1,26-28$ & 101.96 & $\mathrm{~F}$ & & 16 & & 12 & 20 & 18 & 1 & 19 & & 2 & 1 & & & & & 2 & & i & & & & & & & 143 \\
\hline $12 \mathrm{H}-1,43-45$ & 102.13 & $\mathrm{~F}$ & & 34 & 5 & 7 & 26 & 4 & & 36 & 2 & & i & & & & & & & 3 & & & 1 & & & & 182 \\
\hline $12 \mathrm{H}-1,127-129$ & 102.97 & $\mathrm{~F}$ & & 17 & & 5 & 23 & 49 & & 22 & 2 & 9 & i & & & & & 2 & & 3 & 2 & 1 & 4 & & & & 192 \\
\hline $12 \mathrm{H}-2,95-97$ & 104.15 & $\mathrm{~F}$ & 5 & 19 & 1 & 10 & 58 & 52 & 3 & 11 & & 5 & 3 & 1 & 1 & & & 2 & 1 & 1 & 1 & 1 & 20 & & & & 262 \\
\hline $12 \mathrm{H}-3,23-25$ & 104.93 & $\mathrm{~F}$ & 1 & 49 & & 19 & 80 & 21 & 3 & 13 & 1 & 4 & 1 & & & & & I & & 2 & & & 7 & & & & 283 \\
\hline $12 \mathrm{H}-3,43-45$ & 105.13 & $\mathrm{~F}$ & 1 & 14 & & 4 & 37 & 19 & 1 & 16 & & & & & 1 & & & 2 & & 3 & & & & & & & 136 \\
\hline $12 \mathrm{H}-3,63-65$ & 105.33 & $\mathrm{~F}$ & 2 & 34 & & 8 & 51 & 60 & & 35 & & & 1 & & & & & 1 & & & & & 4 & & & & 276 \\
\hline $12 \mathrm{H}-3,85-87$ & 105.55 & $\mathrm{~F}$ & 2 & 5 & & 4 & 22 & 9 & 10 & 10 & & 1 & 2 & & 1 & & & 3 & & & 1 & & & & & & 102 \\
\hline $12 \mathrm{H}-3,105-107$ & 105.75 & $\mathrm{~F}$ & & 19 & & 7 & 55 & 38 & & 30 & & i & 2 & & i & & & 3 & & 2 & & & & & & & 214 \\
\hline $12 \mathrm{H}-3,127-129$ & 105.97 & F & 1 & 63 & & 3 & 54 & 20 & & 62 & & 6 & 14 & & 3 & & & 2 & & & & & & & & & 318 \\
\hline $12 \mathrm{H}-4,55-57$ & 106.75 & $\mathrm{~F}$ & & 25 & & 6 & 16 & 16 & 4 & 11 & & 2 & & & & & & $i$ & & & & & 3 & & & & 124 \\
\hline $12 \mathrm{H}-4,77-79$ & 106.97 & $\mathrm{~F}$ & & 15 & 1 & 8 & 43 & 26 & & 20 & 2 & & & & & & & & & & & & 2 & & & & 167 \\
\hline $12 \mathrm{H}-4,95-97$ & 107.15 & $\mathrm{~F}$ & & 30 & 1 & 8 & 44 & 38 & 1 & 24 & & 2 & 2 & & & & & & & 1 & I & & 14 & & & & 260 \\
\hline $12 \mathrm{H}-4,113-115$ & 107.33 & $\mathrm{~F}$ & & 12 & & 2 & 39 & $\begin{array}{r}30 \\
4\end{array}$ & 1 & $\begin{array}{r}24 \\
7\end{array}$ & & 1 & $1_{1}^{2}$ & & & & & & & $\frac{1}{2}$ & 1 & & $\begin{array}{r}14 \\
8\end{array}$ & & & & 110 \\
\hline $12 \mathrm{H}-4,135-137$ & 107.55 & $\mathrm{~F}$ & & 12 & & 10 & 23 & 11 & & 6 & & & & & & & & 1 & & & & & ${ }_{3}^{\circ}$ & & & & 113 \\
\hline $12 \mathrm{H}-5,5-7$ & 107.75 & $\mathrm{~F}$ & & 17 & & 16 & 52 & 47 & 1 & 47 & 4 & 3 & & & & & & 2 & & 2 & 1 & & & & 2 & 1 & 293 \\
\hline $12 \mathrm{H}-5,23-25$ & 107.93 & $\mathrm{~F}$ & & 12 & & 9 & 39 & 25 & & 18 & 4 & 11 & & & & & & 2 & & & 2 & & & & & & 193 \\
\hline $12 \mathrm{H}-5,43-45$ & 108.13 & $\mathrm{~F}$ & & 16 & & 9 & 19 & 27 & 2 & 24 & & 2 & 1 & & 1 & & & 2 & 2 & 1 & & & 1 & & & & 159 \\
\hline $12 \mathrm{H}-5,66-68$ & 108.36 & $\mathrm{~F}$ & & 8 & 4 & 2 & 15 & 20 & & 10 & 10 & & & & & & & & & & & & 1 & & & & 105 \\
\hline $12 \mathrm{H}-5,83-85$ & 108.53 & $\mathrm{~F}$ & 2 & 11 & 1 & 3 & 25 & 9 & & 15 & & 2 & & & & & 1 & & & & & & & & & & 82 \\
\hline $12 \mathrm{H}-6,13-15$ & 109.33 & $\mathrm{~F}$ & 4 & 29 & & 10 & 44 & 29 & 1 & 54 & 1 & 2 & 3 & & & & & 2 & & & & & & & & & 220 \\
\hline $12 \mathrm{H}-6,34-36$ & 109.54 & $\mathrm{~F}$ & 2 & 27 & 2 & 24 & 56 & 29 & 2 & 40 & 5 & 4 & 1 & 1 & 2 & & & 8 & & & & & & & & & 260 \\
\hline $12 \mathrm{H}-6,55-57$ & 109.75 & $\mathrm{~F}$ & & & & & & & & & & & & & & & & & & & & & & & & & 4 \\
\hline $12 \mathrm{H}-6,73-75$ & 109.93 & $\mathrm{~F}$ & & & & & & & & & & & & & & & & & & & & & & & & & 4 \\
\hline $12 \mathrm{H}-6,95-97$ & 110.15 & $\mathrm{~F}$ & & 5 & & 2 & 16 & 4 & & 4 & & & & & & & & 2 & & & & & & & & & 51 \\
\hline $12 \mathrm{H}-6,113-115$ & 110.33 & $\mathrm{~F}$ & & & & & & & & & & & & & & & & & & & & & & & & & 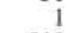 \\
\hline $12 \mathrm{H}-7,5-7$ & 110.75 & F & & 22 & & 29 & 79 & 37 & 2 & 35 & & 7 & 2 & & & & & 4 & & 2 & 1 & 1 & & 1 & & & 313 \\
\hline $12 \mathrm{H}-7,23-25$ & 110.93 & $\mathrm{~F}$ & 1 & 9 & 3 & 19 & 73 & 44 & & 24 & & & 5 & & 1 & & & & & 5 & & & & & & & 231 \\
\hline $12 \mathrm{H}-7,44-46$ & 111.14 & $\mathrm{~F}$ & & & & & & & & & & & & & & & & & & & & & & & & & 6 \\
\hline $12 \mathrm{H}-7,66-68$ & 111.36 & $\mathrm{~F}$ & & & & 1 & & & & & & & & & & & & & & & & & & & & & 8 \\
\hline
\end{tabular}

Note: See Table 1. Abundance $=$ number of specimens 
Table 7. Occurrences and abundance of benthic foraminifers, Hole $887 \mathrm{C}$.

\begin{tabular}{|c|c|c|c|c|c|c|c|c|c|c|c|c|c|c|c|c|c|c|c|c|c|c|c|c|}
\hline $\begin{array}{l}\text { Core, section, } \\
\text { interval }(\mathrm{cm})\end{array}$ & $\begin{array}{l}\text { Depth } \\
\text { (mbsf) }\end{array}$ & 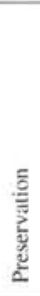 & 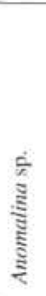 & 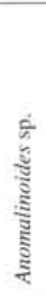 & & 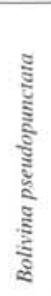 & 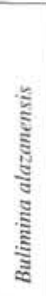 & 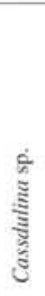 & 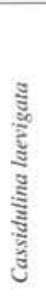 & 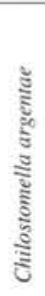 & 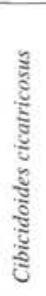 & 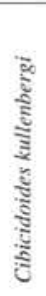 & 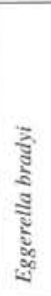 & 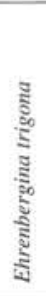 & 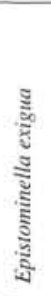 & 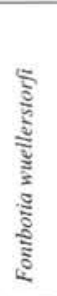 & 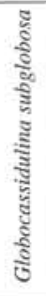 & 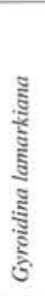 & 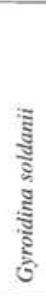 & 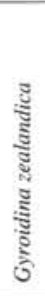 & 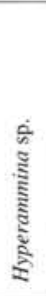 & $\frac{\text { है }}{\text { है }}$ & 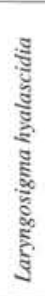 & 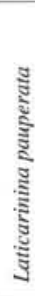 \\
\hline $\begin{array}{l}12 \mathrm{H}-1,5-7 \\
12 \mathrm{H}-1,22-24\end{array}$ & $\begin{array}{l}93.35 \\
93.52\end{array}$ & $\underset{\mathrm{F}}{\mathrm{F}}$ & & & & 1 & & & & & 1 & & 9 & & & 5 & 3 & 3 & 2 & & & 1 & & 4 \\
\hline $12 \mathrm{H}-1,45-47$ & 93.75 & F & & & 2 & & & & & & 5 & 4 & 11 & 1 & 3 & 13 & 2 & & 2 & & & & & 3 \\
\hline $12 \mathrm{H}-1,65-67$ & 93.95 & $\mathrm{~F}$ & & & & & & 1 & & & & & 7 & & & & 2 & & & 1 & & & & 6 \\
\hline $12 \mathrm{H}-1,105-107$ & 94.35 & $\mathrm{~F}$ & & & 1 & & 2 & & & & & 9 & 12 & & 7 & 14 & 5 & & & & & & & 1 \\
\hline $12 \mathrm{H}-1.125-127$ & 94.55 & $\mathrm{~F}$ & & & 3 & & & & & & & & 15 & & 5 & 33 & 3 & 5 & & 1 & & & & 7 \\
\hline $12 \mathrm{H}-1,145-147$ & 94.75 & $\mathrm{~F}$ & & & 5 & & & & & & 1 & 5 & 7 & 2 & 5 & 9 & & & & 4 & & & & 7 \\
\hline $13 \mathrm{H}-1,5-7$ & 102.85 & $\mathrm{~F}$ & & 1 & & & & & & & 2 & & 3 & & 7 & 1 & 17 & 2 & & & 1 & & & i \\
\hline $13 \mathrm{H}-1,25-27$ & 103.05 & $\mathrm{~F}$ & & & 1 & & & & & & & & i & 2 & 10 & 6 & 25 & 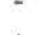 & 5 & 2 & & & & i \\
\hline $13 \mathrm{H}-1.45-47$ & 103.25 & $\mathrm{~F}$ & & & & & & & 1 & & & & 11 & & 1 & & 2 & 1 & 5 & 2 & & & & \\
\hline $13 \mathrm{H}-1,65-67$ & 103.45 & $\mathrm{~F}$ & & & & & & & & & & & 12 & & 2 & 1 & 7 & i & & & & & & \\
\hline $13 \mathrm{H}-1,85-87$ & 103.65 & $\mathrm{~F}$ & & & & & & & & 2 & 1 & & 15 & & 5 & 11 & 53 & 4 & & 1 & & 3 & & \\
\hline $13 \mathrm{H}-1,105-107$ & 103.85 & $\mathrm{~F}$ & & & & & & & & & & & 6 & 1 & 3 & 1 & 9 & 4 & & & & & & 2 \\
\hline $13 \mathrm{H}-1,125-127$ & 104.05 & $\mathrm{~F}$ & 1 & & 8 & & & & & 1 & & 5 & 11 & 2 & 24 & 16 & 49 & 3 & & & & & & 4 \\
\hline $13 \mathrm{H}-1,145-147$ & 104.25 & $\mathrm{~F}$ & & & 4 & & & & & & & & 17 & 1 & 30 & 6 & 35 & & 6 & & & & 1 & 1 \\
\hline
\end{tabular}
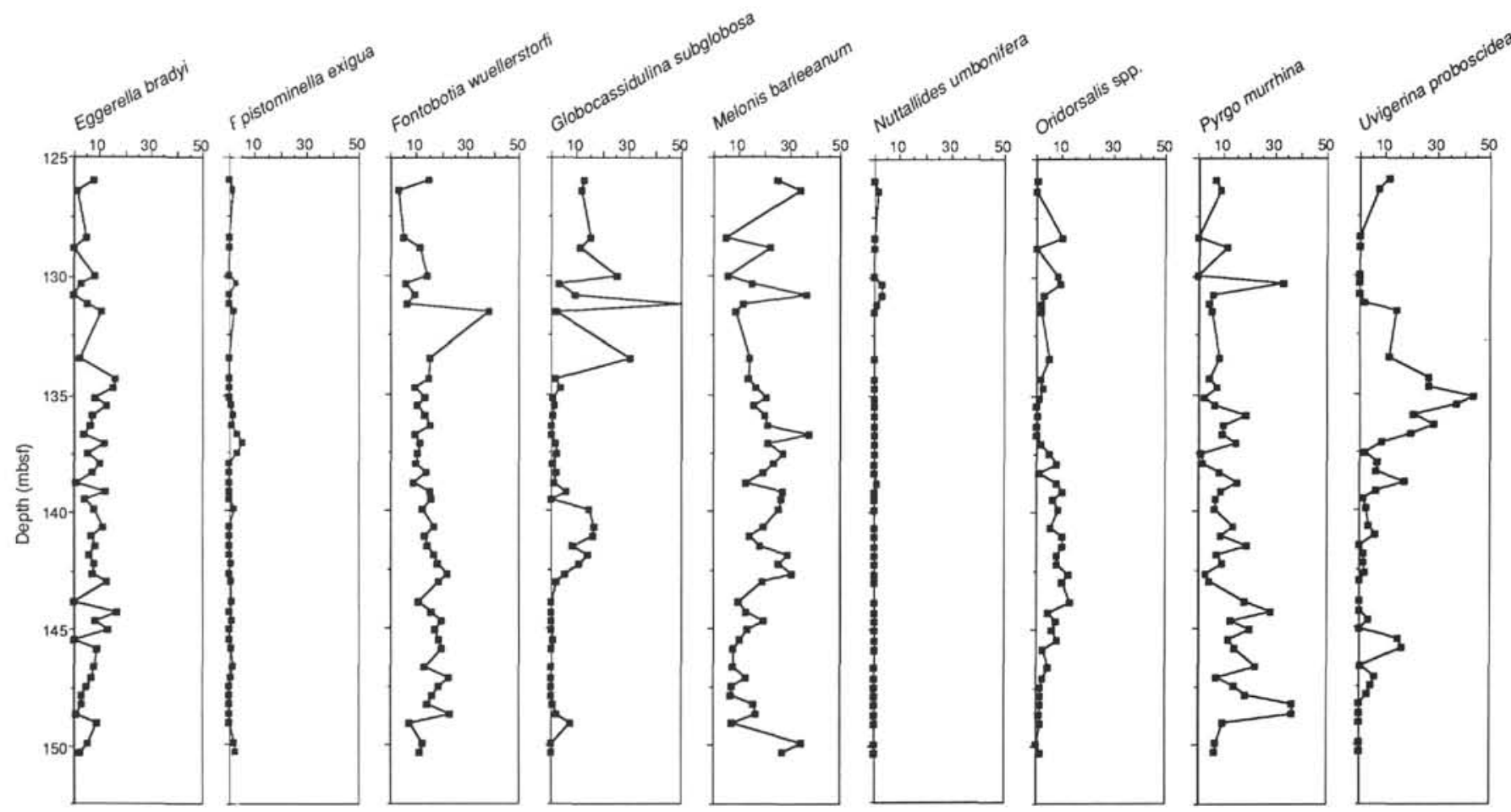

Figure 3. Percent abundance of selected benthic foraminifers vs. depth for Hole 883B (Oridorsalis spp. = Oridorsalis tener [Brady] and Oridorsalis umbonatus [Reuss]).

They noted that it is a highly variable species with four to five large chambers in the final whorl, reticulate microcrystalline and crystalline ultrastructure related to secondary calcification, and large apertural variability including the occasional development of an apertural lip. They indicated that small forms of $N$. atlantica with a kummerform final chamber intergrade morphologically with Neogloboquadrina pachyderma.

Poore (1981) documented the presence of $N$. atlantica in the foraminifer assemblages of DSDP Sites $467-471$ in the North Pacific
Ocean. Aside from being predominantly dextrally coiled, these specimens are essentially identical to those observed in the North Atlantic.

At DSDP Site 552, Dowsett and Poore (1990) noted that encrustation of tests sometimes made the distinction between Globigerina bulloides and Neogloboquadrina atlantica difficult. We find that the amount of secondary calcification occurring in many samples from Sites 883 and 887 makes it extremely difficult to distinguish between these two taxa. Plate 2 (Fig. 10) shows a Globigerina bulloides specimen showing recalcification of all but the ultimate kummerform 
Table 7 (continued).

\begin{tabular}{|c|c|c|c|c|c|c|c|c|c|c|c|c|c|c|c|c|c|c|c|c|c|c|c|c|c|}
\hline $\begin{array}{l}\text { Core, section, } \\
\text { interval }(\mathrm{cm})\end{array}$ & $\begin{array}{l}\text { Depth } \\
\text { (mbsf) }\end{array}$ & 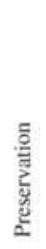 & 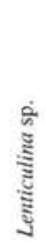 & 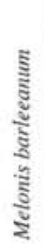 & 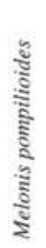 & 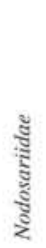 & 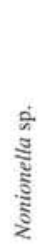 & 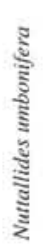 & 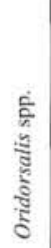 & 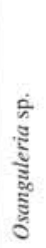 & 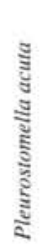 & 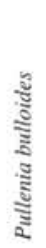 & 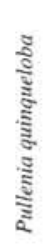 & 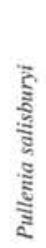 & $\begin{array}{l}\hat{\mathrm{C}} \\
\hat{5} \\
\frac{8}{2} \\
2\end{array}$ & 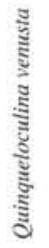 & 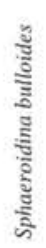 & 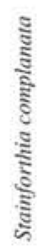 & 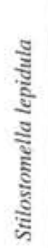 & 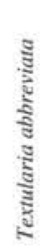 & 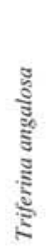 & 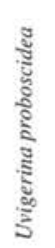 & $\begin{array}{l}\frac{\dot{0}}{6} \\
\frac{g}{5} \\
\frac{3}{3} \\
\frac{3}{3}\end{array}$ & 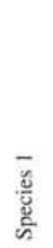 & Total \\
\hline $\begin{array}{l}12 \mathrm{H}-1.5-7 \\
12 \mathrm{H}-1.22-24\end{array}$ & $\begin{array}{l}93.35 \\
93.52\end{array}$ & $\begin{array}{l}\mathrm{F} \\
\mathrm{F}\end{array}$ & 2 & 3 & & 10 & & 24 & & & & 2 & & 1 & & & & & 1 & & & 18 & & 1 & $\begin{array}{r}91 \\
2\end{array}$ \\
\hline $12 \mathrm{H}-1,45-47$ & 93.75 & $\mathrm{~F}$ & & 32 & 1 & 7 & & 29 & 6 & & & 5 & & 1 & & & & & & 1 & & 1 & & & 129 \\
\hline $12 \mathrm{H}-1,65-67$ & 93.95 & $\mathrm{~F}$ & & 7 & & 6 & & 38 & 2 & & & & & 2 & & & & & 3 & & & 17 & & & 92 \\
\hline $12 \mathrm{H}-1,105-107$ & 94.35 & $\mathrm{~F}$ & 1 & 20 & 1 & 9 & & 47 & 3 & & & 14 & & 2 & & & & 1 & 3 & & & ii & & & 163 \\
\hline $12 \mathrm{H}-\mathrm{I}, 125-127$ & 94.55 & $\mathrm{~F}$ & & 25 & & 15 & & 92 & 29 & & & 16 & 1 & & & 2 & & & & & & 22 & 2 & & 276 \\
\hline $12 \mathrm{H}-1,145-147$ & 94.75 & $\mathrm{~F}$ & & 41 & & 9 & & 42 & 12 & 1 & 4 & 8 & & & 2 & 1 & & & 3 & & & 25 & 3 & & 196 \\
\hline $13 \mathrm{H}-1,5-7$ & 102.85 & $\mathrm{~F}$ & & 6 & & 5 & & 15 & 15 & & & 18 & & 2 & $i$ & & 2 & 1 & & & & & & & 100 \\
\hline $13 \mathrm{H}-1,25-27$ & 103.05 & $\mathrm{~F}$ & & 19 & & 3 & & 22 & 15 & & 1 & 14 & & & 4 & & 2 & & & & 1 & 1 & & & 135 \\
\hline $13 \mathrm{H}-1,45-47$ & 103.25 & $\mathrm{~F}$ & & 2 & 2 & 1 & & 4 & 1 & & & 7 & & 1 & i & & & & & & & & & & 35 \\
\hline $13 \mathrm{H}-1,65-67$ & 103.45 & $\mathrm{~F}$ & & $\overline{3}$ & & 4 & & 12 & 3 & & & 4 & & & 2 & & & 1 & & & & & & & 52 \\
\hline $13 \mathrm{H}-1,85-87$ & 103.65 & $\mathrm{~F}$ & & 14 & 1 & 13 & 2 & 37 & 10 & & 2 & 14 & 2 & & & & & & & & & 6 & & & 196 \\
\hline $13 \mathrm{H}-1,105-107$ & 103.85 & $\mathrm{~F}$ & & & 4 & & & 15 & 4 & & & 7 & 1 & 11 & 2 & & 2 & & & & & & & & 72 \\
\hline $13 \mathrm{H}-1,125-127$ & 104.05 & $\mathrm{~F}$ & & 32 & & 24 & 1 & 67 & 51 & & 2 & 41 & 7 & & 6 & & 1 & & 1 & & & 11 & & & 368 \\
\hline $13 \mathrm{H}-1,145-147$ & 104.25 & $\mathrm{~F}$ & & 17 & & 5 & & 50 & 25 & & & 16 & & 1 & 1 & & i & & & & 1 & 13 & & & 231 \\
\hline
\end{tabular}

Note: See Table 1. Abundance $=$ number of specimens.

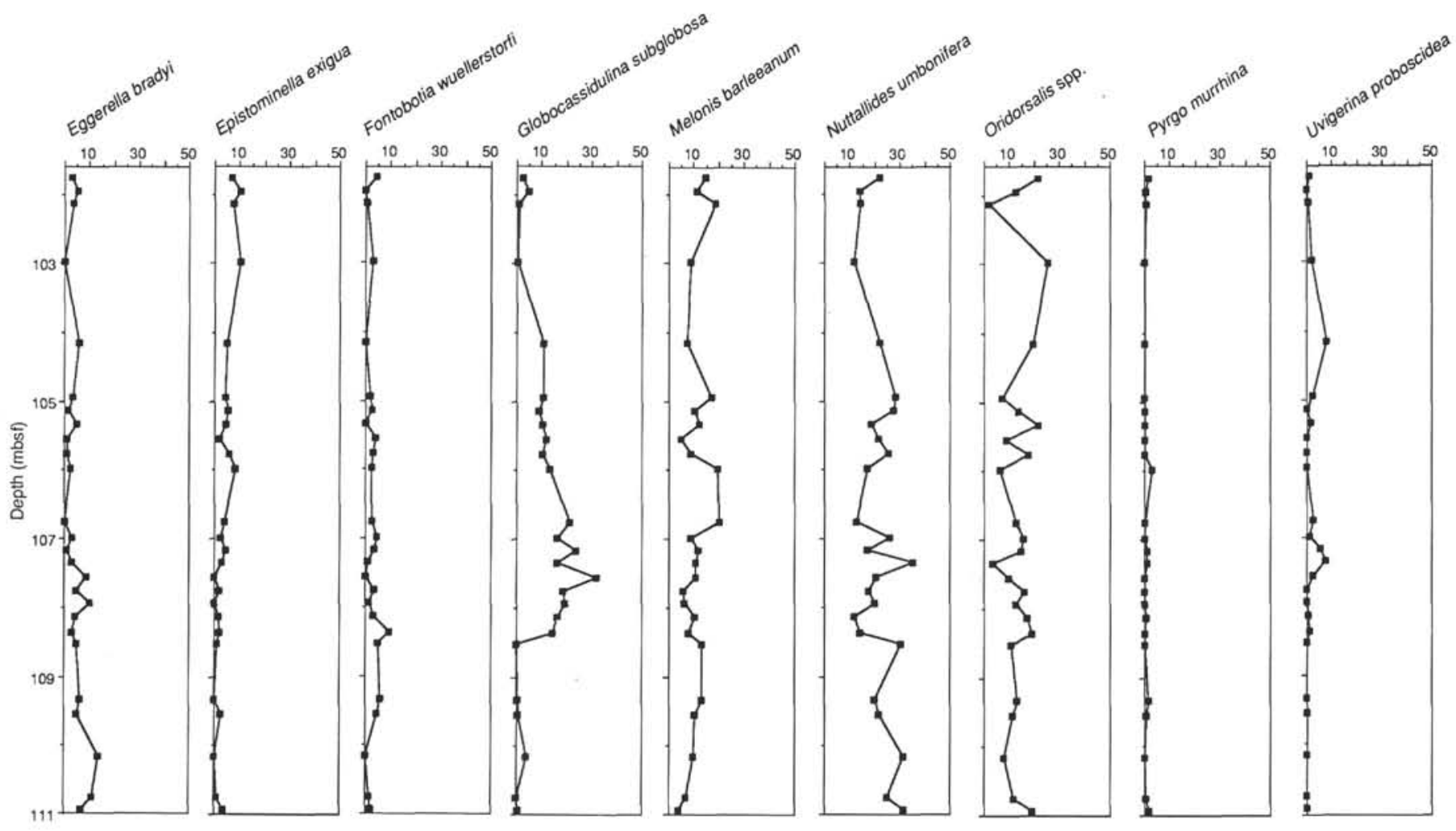

Figure 4. Percent abundance of selected benthic foraminifers vs. depth for Hole 887A (Oridorsalis spp. = Oridorsalis tener [Brady] and Oridorsalis umbonatus [Reuss]).

chamber. This obliterates the distinctive globigerine surface ultrastructure shown in Plate 2 (Fig. 12). Plate 3 (Figs. 1 and 2) shows the reticulate microcrystalline surface ultrastructure with pore pits typical of Neogloboquadrina spp. Fully crystalline structure can be found on many specimens from Sites 883 and 887.

No other workers have reported the presence of $N$. atlantica in the Pliocene assemblages of the subarctic North Pacific. We agree with Poore (1981) that $N$. pachyderma form 3 of Keller (1978a, 1978b), documented at DSDP Sites 173 and 310 and the Centerville Beach section of northern California (Keller and Ingle, 1981) as well as Neogloboquadrina asanoi (Maiya, Saito, and Sato) and Neogloboquadrina kagaensis (Maiya, Saito, and Sato), are referable to $N$. atlantica. We postulate that at higher latitudes, $N$. atlantica has been previously identified as Globigerina bulloides or Neogloboquadrina pachyderma, as a result of poor preservation and crystalline overgrowth. 


\section{Mid-Pliocene North Pacific Sea-surface Conditions}

The lack of a sufficient number of high-latitude coretop carbonate-bearing samples for temperature calibration preclude the use of transfer functions or quantitative modern analog techniques of temperature estimation. However, late Pleistocene sequences from DSDP Legs 18 and 19 provide a semiquantitative summary of the planktonic foraminifer fauna near the position of ODP Sites 883 and 887 . The late Pleistocene faunas are similar from these two regions and are similar to the Pliocene fauna recovered from ODP Site 883. This suggests that the Oyashio Current and Western Subarctic Gyre were in the same approximate positions during the middle Pliocene as they are today. The high-frequency variability seen in the Site 883 assemblages may indicate periodic migration of the path of the Oyashio bringing cooler Arctic water closer to Site 883. Additional work on the Site 883 material is necessary to improve the age model and sampling interval so as to address this question fully.

The Pliocene planktonic assemblages recovered from Site 887 generally show increased numbers of warmer taxa than have been recovered from Pleistocene DSDP Leg 18 material. A similar fauna dominated by $G$. bulloides is recorded by Lagoe et al. (1993) from the Yakataga Formation in the Gulf of Alaska. Barron (1992) documented warmer than modern conditions at DSDP Site 580 that coincided with warming documented in the North Atlantic by Dowsett and Poore (1991). Similar warming has been documented at DSDP Site 36 by Poore (in press). This warming in the North Pacific during the middle Pliocene most likely represents increased heat transport to the Eastern Pacific by way of the Kuroshio Current system.

\section{Mid-Pliocene North Pacific Deep-water Conditions}

The benthic foraminifer faunas described from Sites 883 and 887 represent distinct depth-related faunas. The Site 887 fauna shows relative stability and represents a deep-water, upper abyssal (2000$4000 \mathrm{~m}$ ) fauna thus indicating no significant late Pliocene deep water change in the North Pacific. The fauna described from Site 883 represents a shallower water setting (lower bathyal) and shows greater variability in species abundance than the Site 887 fauna. A significant shift occurs at Site 883, with an increase in Uvigerina proboscidea indicating a deepening of the oxygen minima and marking a transition from a Melonis barleeanum-dominated assemblage to a Globocassidulina subglobosa dominated assemblage. We suggest a significant intermediate to upper deep water change represented by a deepening of the oxygen minimum in the North Pacific, possibly related to a global oceanographic change that also affected the preservation of $\mathrm{CaCO}_{3}$ in the Pacific basin at about 2.9 Ma (Farrell and Prell, 1991).

\section{SUMMARY}

This preliminary study indicates that the mid-Pliocene foraminifer faunas at Sites 883 and 887 can be analyzed to estimate paleoceanographic and paleoclimatic conditions in the subarctic North Pacific.

No evidence for mid-Pliocene sea-surface warming can be found at Site 883; however, minor warming is associated with the planktonic faunas at Site 887. This warming is evident in the Yakataga Formation of Alaska and as far south as DSDP Site 36. This implies a greater transport of heat by way of the Kuroshio system during the midPliocene. A look at the longer record from Site 887 may reveal a deep-water record of oceanographic changes associated with other climatic fluctuations recorded in the Yakataga Formation.

The planktonic assemblages at Sites 883 and 887 contain Neogloboquadrina atlantica (Berggren) and firmly establish the presence of this taxon in the high-latitude North Pacific. Because of the difficulty in separating some specimens of $N$. atlantica from recalcified specimens of Globigerina bulloides using light microscopy, extreme caution should be used when either of these taxa are incorporated into paleoenvironmental studies.

\section{ACKNOWLEDGMENTS}

We thank J. Barron, R.Z. Poore, and T.M. Cronin for their thoughtful reviews of this manuscript. E. Polanco, J. Self-Trail, and M. Robinson provided excellent laboratory assistance. We thank the Ocean Drilling Program for making the samples available for this study.

\section{REFERENCES $*$}

Barron, J.A., 1992. Pliocene paleoclimatic interpretations of DSDP Site 580 (NW Pacific) using diatoms. Mar. Micropaleontol., 20:23-44.

Berggren, W.A., Kent, D.V., and Van Couvering, J.A., 1985. The Neogene: Part 2. Neogene geochronology and chronostratigraphy. In Snelling, N.J. (Ed.), The Chronology of the Geological Record. Geol. Soc. London Mem., 10:211-260.

Blow, W.H., 1969. Late middle Eocene to Recent planktonic foraminiferal biostratigraphy. In Brönniman, P., and Renz, H.H. (Eds.), Proc. First Int. Conf. Planktonic Microfossils, Geneva, 1967: Leiden (E.J. Brill), 1:199422.

Cande, S.C., and Kent, D.V., 1992. A new geomagnetic polarity time scale for the Late Cretaceous and Cenozoic. J. Geophys. Res., 97:13917-13951.

Cronin, T.M., and Dowsett, H.J., 1993. PRISM: warm climates of the Pliocene. Geotimes, 38:17-19.

Cronin, T.M, Whatley, R.C., Wood, A., Tsukagoshi, A., Ikeya, N., Brouwers, E.M., and Briggs, W.M., Jr., 1993. Microfaunal evidence for elevated mid-Pliocene temperatures in the Arctic Ocean. Paleoceanography, 8:161-173.

Dowsett, H.J., and Cronin, T.M., 1990. High eustatic sea level during the middle Pliocene: evidence from the southeastern U.S. Atlantic Coastal Plain. Geology, 18:435-438.

Dowsett, H.J., Cronin, T.M., Poore, R.Z., Thompson, R.S., Whatley, R.C., and Wood, A.M., 1992. Micropaleontological evidence for increased meridional heat transport in the North Atlantic Ocean during the Pliocene. Science, 258:1133-1135.

Dowsett, H.J., and Poore, R.Z., 1990. A new planktic foraminifer transfer function for estimating Pliocene-Holocene paleoceanographic conditions in the North Atlantic. Mar: Micropaleontol., 16:1-23.

1991. Pliocene sea surface temperatures of the North Atlantic Ocean at 3.0 Ma. Quat. Sci. Rev., 10:189-204.

Dowsett, H.J., Thompson, R.S., Barron, J.A., Cronin, T.M., Fleming, R.F., Ishman, S.E., Poore, R.Z., Willard, D.A., and Holtz, T.R., 1994. Joint investigations of the middle Pliocene climate I: PRISM paleoenvironmental reconstructions. Global Planet. Change (Palaeogeogr., Palaeoclimatol., Palaeoecol.), 9:1-54.

Echols, R.J., 1973. Foraminifera, Leg 19, Deep Sea Drilling Project. In Creager, J.S., Scholl, D.W., et al., Init. Repts. DSDP, 19: Washington (U.S. Govt. Printing Office), 721-733.

Echols, R.J., and Armentrout, J.M., 1980. Holocene foraminiferal distribution patterns on the shelf and slope, Yakataga-Yakutat area, northern Gulf of Alaska. In Field, M.E., Bouma, A.H., Colburn, I.P., Douglas, R.G., and Ingle, J.C. (Eds.), Quaternary Depositional Environments of the Pacific Coast. Pacific Coast Paleogeogr. Symp. 4, Soc. Econ. Paleontol. Mineral., 281-303.

Farrell, J.W., and Prell, W.L., 1991. Pacific $\mathrm{CaCO}_{3}$ preservation and $\delta^{18} \mathrm{O}$ since 4 Ma: paleoceanic and paleoclimatic implications. Paleoceanography, 6:485-498.

Ingle, J.C., Jr., 1973. Neogene foraminifera from the northeastern Pacific Ocean, Leg 18, Deep Sea Drilling Project. In Kulm, L.D., von Huene, R., et al., Init. Repts. DSDP, 18: Washington (U.S. Govt. Printing Office), $517-567$.

Ingle, J.C., Jr., and Keller, G., 1980. Benthic foraminiferal biofacies of the eastern Pacific margin between $40^{\circ} \mathrm{S}$ and $32^{\circ} \mathrm{N}$. In Field, M.E., Bouma, A.H., Colburn, I.P., Douglas, R.G., and Ingle, J.C. (Eds.), Quaternary Depositional Environments of the Pacific Coast. Pacific Coast Paleogeogr. Symp. 4, Soc. Econ. Paleontol. Mineral., 341-355.

Keigwin, L.D., Jones, G.A., and Froelich, N.P., 1992. A 15,000-year paleoenvironmental record from Meiji seamount, far northwest Pacific. Earth Planet Sci. Lett., 111:425-440.

\footnotetext{
-Abbreviations for names of organizations and publications in ODP reference lists follow the style given in Chemical Abstracts Service Source Index (published by American Chemical Society).
} 
Keller, G., 1978a. Late Neogene biostratigraphy and paleoceanography of DSDP Site 310 Central North Pacific and correlation with the Southwest Pacific. Mar. Micropaleontol., 3:97-119.

1978b. Morphologic variation of Neogloboquadrina pachyderme (Ehrenberg) in sediments of the marginal and central northeast Pacific Ocean and paleoclimatic interpretation. J. Foraminiferal Res., 8:208-224.

Keller, G., and Ingle, J.C., 1981. Planktonic foraminiferal biostratigraphy, paleoceanographic implications, and deep-sea correlation of the PliocenePleistocene Centerville Beach section, northern California. Spec. Pap.Geol. Soc. Am., 184:127-135.

Kennett, J.P., and Srinivasan, M.S., 1983. Neogene Planktonic Foraminifera: A Phylogenetic Atlas: Stroudsburg, PA (Hutchinson Ross).

Lagoe, M.B., Eyles, C.H., Eyles, N., and Hale, C., 1993. Timing of late Cenozoic tidewater glaciation in the far North Pacific. Geol. Soc. Am. Bull., 105:1542-1560.

Lagoe, M.B., and Thompson, P.R., 1988. Chronostratigraphic significance of late Cenozoic planktonic foraminifera from the Ventura Basin, California: potential for improving tectonic and depositional interpretation. J. Foraminiferal Res., 18:250-266.

Loeblich, A.R., Jr., and Tappan, H., 1988. Foraminiferal Genera and their Classification: New York (Van Nostrand Reinhold).

Murray, J.W., 1991. Ecology and Palaeoecology of Benthic Foraminifera: London (Longman).

Parker, F.L., 1962. Planktonic foraminiferal species in Pacific sediments. Micropaleontology, 8:219-254.

1967. Late Tertiary biostratigraphy (planktonic foraminifera) of tropical Indo-Pacific deep-sea cores. Bull. Am. Paleontol., 52:111-208.

Poore, R.Z., 1981. Miocene through Quaternary planktonic foraminifers from offshore southern California and Baja California. In Yeats, R.S., Haq, B.U., et al., Init. Repts. DSDP, 63: Washington (U.S. Govt. Printing Office), $415-436$.

, in press. Mid-Pliocene planktic foraminifers and environmental estimates from northeastern Pacific DSDP Site 36. In Wrenn, J., and Suc, J.P. (Eds.), Pliocene Climates. Am. Assoc. Stratigr. Palynol.

Poore, R.Z., and Berggren, W.A., 1975. The morphology and classification of Neogloboquadrina atlantica (Berggren). J. Foraminiferal Res., 5:76-84.

Schweitzer, P.N., 1993. Modern average global sea-surface temperature. U.S. Geol. Surv. Digital Data Ser., DDS-10.

Shackleton, N.J., Hall, M.A., and Pate, D., in press. Pliocene stable isotope stratigraphy of Site 846. In Pisias, N.G., Mayer, L.A., Janecek, T.R., Palmer-Julson, A., and van Andel, T.H. (Eds.), Proc. ODP, Sci. Results, 138: College Station, TX (Ocean Drilling Program).

Thomas, E., 1985. Late Eocene to Recent deep-sea benthic foraminifers from the central equatorial Pacific Ocean. In Mayer, L., Theyer, F., Thomas, E., et al., Init. Repts. DSDP, 85: Washington (U.S. Govt. Printing Office), 655-694.

Webb, P.-N., and Harwood, D.M., 1991. Late Cenozoic glacial history of the Ross Embayment, Antarctica. Quat. Sci. Rev., 10:215-223.

Willard, D.A., 1994. Palynological record from the North Atlantic Region at $3 \mathrm{Ma}$ : vegetational response to a period of global warmth. Rev. Palaeobot. Palynol., 83:275-297.

Date of initial receipt: 5 April 1994

Date of acceptance: 10 August 1994

Ms 145SR-150 


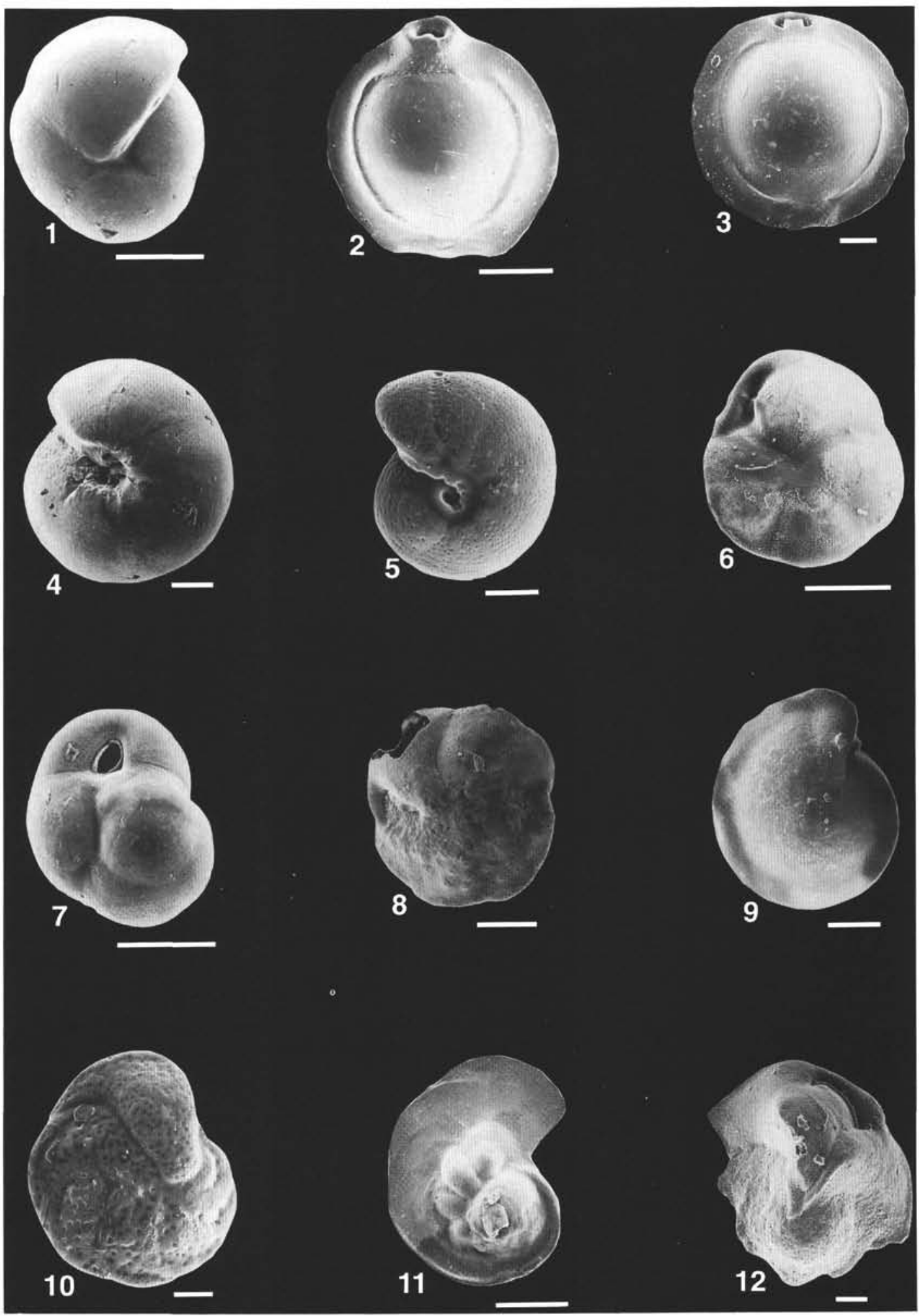

Plate 1. 1. Pullenia bulloides (d'Orbigny); Sample 145-883B-16H-4, 35-40 cm; USNM 483280 . 2. Pyrgo murrhina (Schwager); Sample 145-887C-15H-3, 102-107 cm; USNM 483281. 3. Pyrgo depressa (d'Orbigny); Sample 145-887C-15H-3, 102-107 cm; USNM 483282. 4. Gyroidina zealandica Finlay; Sample 145-887C-12H-1, 105-107 cm; USNM 483283. 5. Melonis barleeanum (Williamson); Sample 145-883B-16H-4, 35-40 cm; USNM 483284. 6. Epistominella exigua (Brady); Sample 145-887C-12H-1, 45-47 cm; USNM 483285. 7. Globocassidulina subglobosa (Brady); Sample 145-887A-12H-7, 5-7 cm; USNM 483286. 8. Nuttallides umbonifera (Cushman); Sample 145-887A-12H-7,5-7 cm; USNM 483287. 9. Cibicidoides kullenbergi (Parker); Sample 145-887A-12H-3, 63-65 cm; USNM 483288. 10. Fontbotia wuellerstorfi (Schwager); Sample 145-883B-16H-4, 35-40 cm; USNM 483289. 11. Laticarinina pauperata (Parker and Jones); Sample 145-887A-12H-1, 43-45 cm; USNM 483290. 12. Ehrenbergina trigona Goës; Sample 145-887A-12H-1, 26-28 cm; USNM 483291. Scale bar $=100 \mu \mathrm{m}$. 


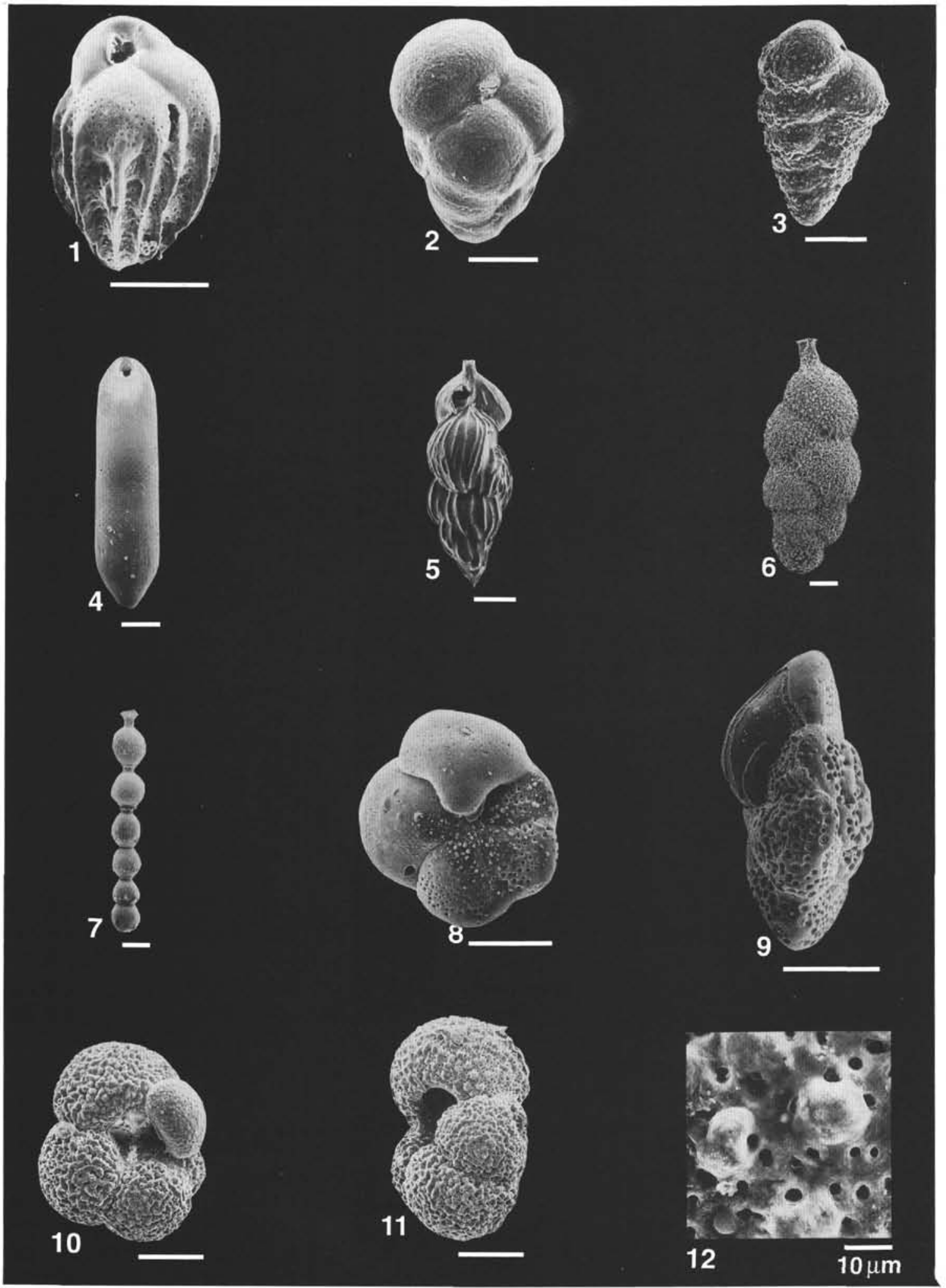

Plate 2. 1. Bulimina alazanensis Cushman; Sample 145-887C-12H-1, 105-107 cm; USNM 483292. 2. Eggerella bradyi (Cushman); Sample 145-887A-12H-1, 43-45 cm; USNM 483293. 3. Bolivina decussata Brady; Sample 145-887A-12H-6, 13-15 cm; USNM 483294. 4. Pleurostomella acuta Hantken; Sample 145-887A-12H-4, 95-97 cm; USNM 483295. 5. Trifarina angulosa (Williamson); Sample 145-883B-16H-4, 35-40 cm; USNM 483296. 6. Uvigerina proboscidea Schwager; Sample 145-887C-15H-3, 102-107 cm; USNM 483297. 7. Stilostommella lepidula (Schwager); Sample 145-887A-12H-1, 43-45 cm; USNM 483298. 8. Globorotalia scitula (Brady); Sample 145-887C-13H-1,5-7 cm; USNM 483299. 9. Globorotalia scitula (Brady); Sample 145-883B-14H-6, 55-60 cm; USNM 483300. 10. Globigerina bulloides d'Orbigny; Sample 145-883B-14H-2, 15-20 cm; USNM 483301. 11. Globigerina bulloides d'Orbigny; Sample 145-887C-12H-1, 125-127 cm; USNM 483302. 12. Detail of ultimate chamber of Figure 11 showing globigerine test surface ultrastructure of pore pitted surface and pustules. Scale bar $=100 \mu \mathrm{m}$ unless otherwise noted. 

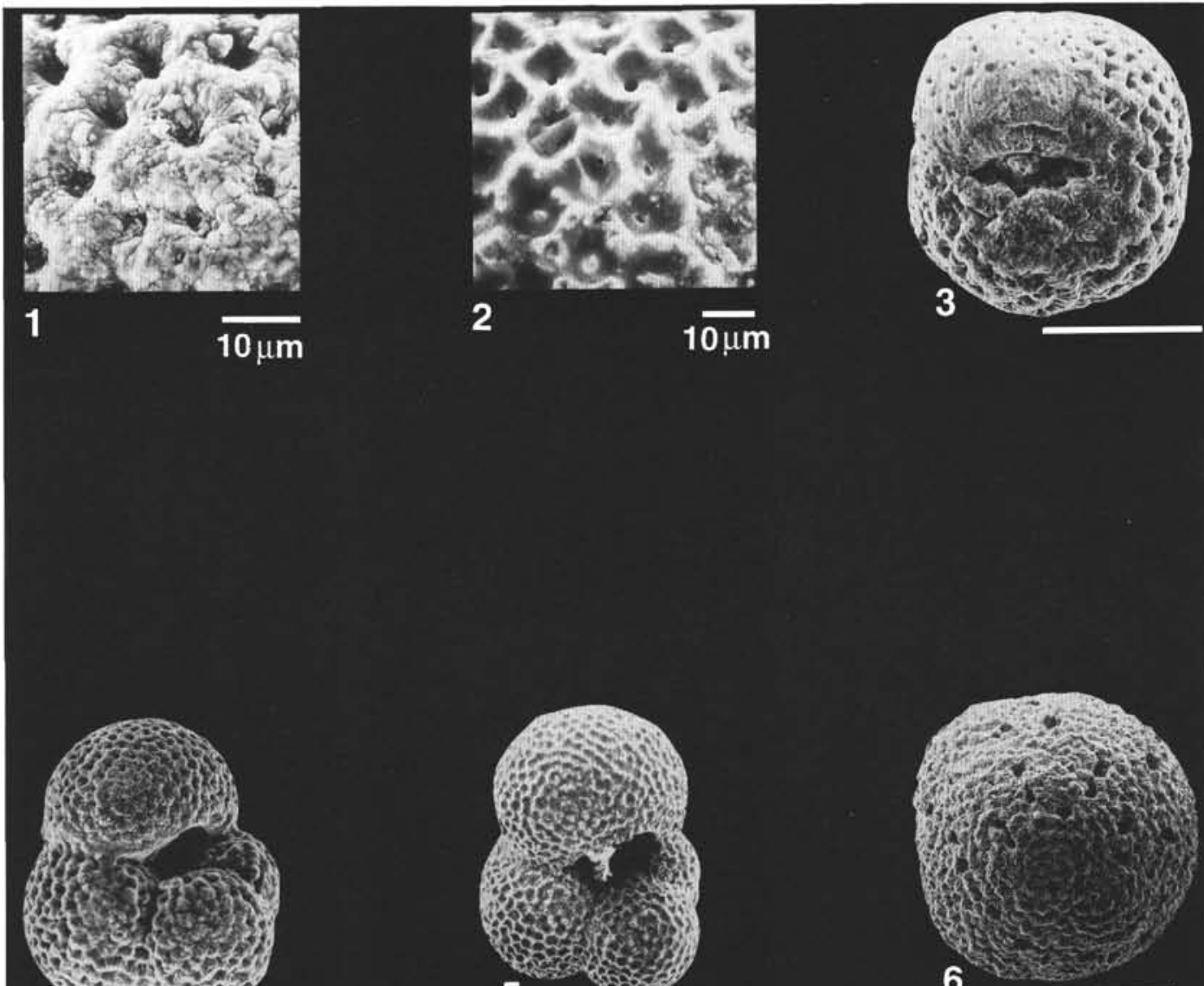

4
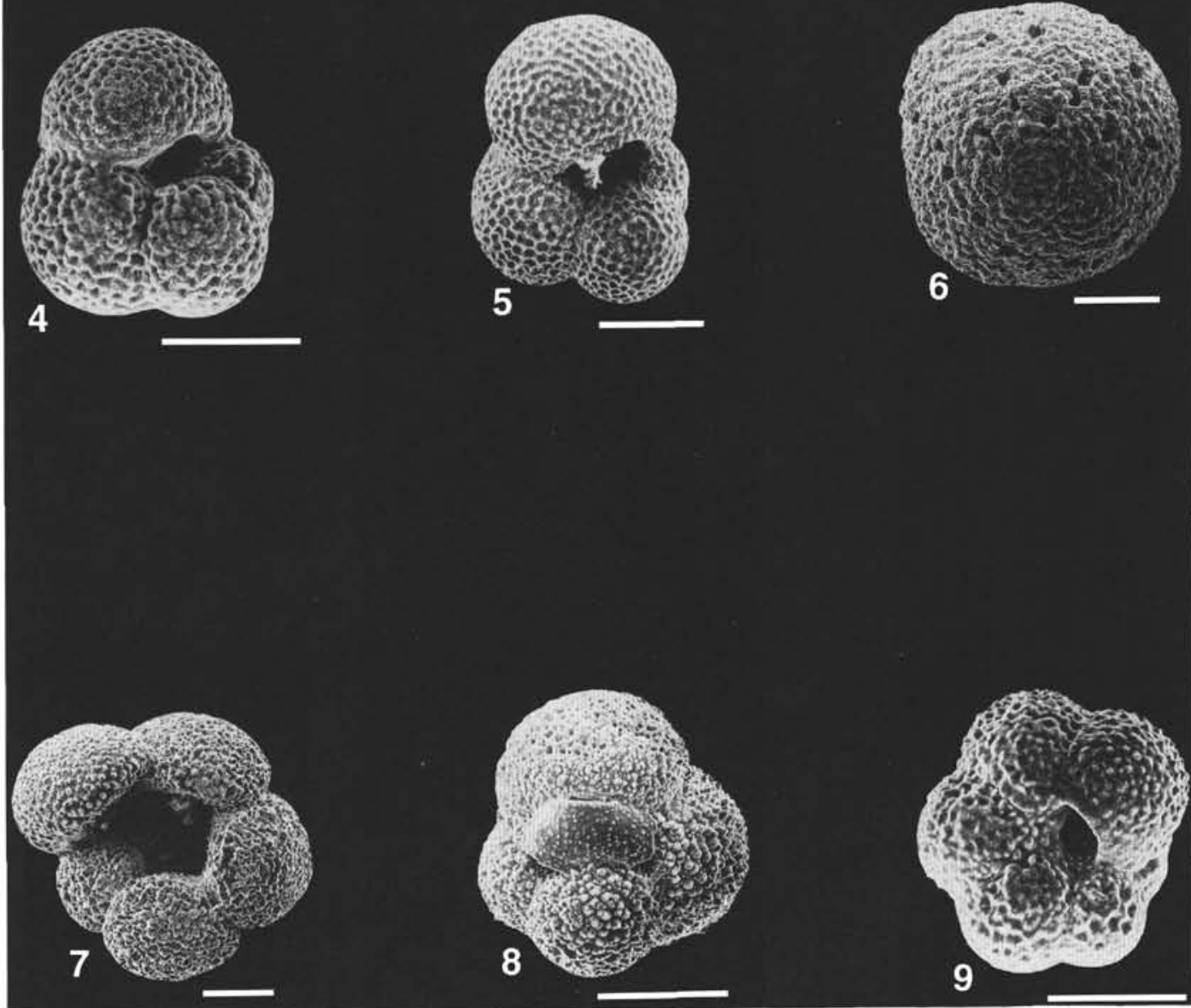

Plate 3. 1. Detail of reticulate-microcrystalline test surface ultrastructure of ultimate chamber of Figure 4. 2. Detail of reticulate-microcrystalline test surface ultrastructure of ultimate chamber of Figure 5. 3. Neogloboquadrina pachyderma (Ehrenberg); Sample 145-883B-14H-2, 15-20 cm; USNM 483303. 4. Neogloboquadrina atlantica (Berggren); Sample 145-883B-14H-2, 15-20 cm; USNM 483304. 5. Neogloboquadrina atlantica (Berggren); Sample 145-887A-12H-3, 105$107 \mathrm{~cm}$; USNM 483305. 6. Orbulina universa d'Orbigny; Sample 145-887A-12H-6, 34-36 cm; USNM 483306. 7. Globigerina umbilicata Orr and Zaitzeff; Sample 145-887A-12H-3,127-129 cm; USNM 483307. 8. Globigerinita glutinata (Egger); Sample 145-883B-15H-7, 35-40 cm; USNM 483308. 9. Turborotalita quinqueloba (Natland); Sample 145-883B$14 \mathrm{H}-1,5-10 \mathrm{~cm}$; USNM 483309. Scale bar $=100 \mu \mathrm{m}$ unless otherwise noted. 